\title{
Luminous AGB stars in nearby galaxies
}

\section{A study using virtual observatory tools ${ }^{\star}$}

\author{
P. Tsalmantza ${ }^{1}$, E. Kontizas ${ }^{2}$, L. Cambrésy ${ }^{3}$, F. Genova ${ }^{3}$, A. Dapergolas ${ }^{2}$, and M. Kontizas ${ }^{1}$
}

\footnotetext{
1 Department of Astrophysics Astronomy \& Mechanics, Faculty of Physics, University of Athens, 15783 Athens, Greece e-mail: vivitsal@phys.uoa.gr

2 Institute for Astronomy and Astrophysics, National Observatory of Athens, PO Box 20048, 11810 Athens, Greece

3 Observatoire Astronomique de Strasbourg, 67000 Strasbourg, France
}

Received 28 March 2005 / Accepted 20 September 2005

\section{ABSTRACT}

Aims. This study focuses on very luminous $\left(M_{\mathrm{bol}}<-6.0 \mathrm{mag}\right)$ AGB stars with $J-K_{\mathrm{s}}>1.5 \mathrm{mag}$ and $H-K_{\mathrm{s}}>0.4 \mathrm{mag}$ in the LMC, SMC, M 31, and M 33 from 2MASS data.

Methods. The data were taken from the 2MASS All-Sky Point Source catalogue archive. We used Virtual Observatory tools and took advantage of its capabilities at various stages in the analysis.

Results. It is well known that stars with the colors we selected correspond mainly to carbon stars. Although the most luminous AGBs detected here contain a large number of carbon stars, they are not included in existing catalogues produced from data in the optical domain, where they are not visible since they are dust-enshrouded. A comparison of the AGB stars detected with combined near and mid-infrared data from MSX and 2MASS in the LMC shows that $10 \%$ of the bright AGB stars are bright carbon stars never detected before and that the other $50 \%$ are OH/IR oxygen rich stars, whereas the $40 \%$ that remain were not cross-matched.

Conclusions. The catalogues of the most luminous AGB stars compiled here are an important complement to existing data. In the LMC, these bright AGB stars are centrally located, whereas they are concentrated in an active star-formation ring in M 31. In the SMC and M 33, there are not enough of them to draw definite conclusions, although they tend to be centrally located. Their luminosity functions are similar for the four galaxies we studied.

Key words. stars: carbon - Local Group - stars: AGB and post-AGB - infrared: stars - Magellanic Clouds

\section{Introduction}

The very red AGB stars, such as the various types of carbon and variable stars in galaxies, trace the intermediate-mass stellar population, thereby providing information about stellar evolution theories. Their spatial distribution is related to the star-formation history of the parent galaxy. These reddest and most extreme AGB stars cannot be detected easily at the conventional $B, R$ wavelengths but can be observed in the near-infrared.

Carbon stars are detected in the optical domain if their mass is less than 4-5 $M_{\odot}$, whereas the more massive ones are expected to be seen only at longer wavelengths. Dredgeup and mass-loss determine whether an AGB star will become a carbon star. Very bright carbon stars can still be produced from stars with masses of 6 or $8 M_{\odot}$, if the mass-loss rate is not too high to allow enough dredge-up episodes (Frost et al. 1998). Models of stars up to $6 M_{\odot}$ for solar and Magellanic metallicities have shown that all massive carbon stars are, or

* Appendix A is only available in electronic form at http://www.edpsciences.org could be, dust-enshrouded and should therefore not be visible at $B$ and $R$ wavelengths (Frost et al. 1998). In the nearinfrared domain, where these stars are revealed best, carbon stars mainly populate the red tail (Nikolaev \& Wenberg 2000; Cioni et al. 2004), which is easily detected in the colormagnitude diagram $M_{K_{\mathrm{s}}}$ vs. $J-K_{\mathrm{s}}$ as an inclined branch of stars departing from an almost vertical sequence of red giants. It is not yet clear whether all stars in that area of the colormagnitude diagram are carbon stars. Marigo et al. (2003) point out that the majority of red tail stars are carbon, and Davidge (2003) showed that, in NGC 205, carbon stars are those among the red tail with the additional constraints $J-K_{\mathrm{s}}>1.5 \mathrm{mag}$ and $H-K_{\mathrm{s}}>0.4 \mathrm{mag}$.

Adopting the above criteria for carbon stars, we selected carbon star candidates in the Magellanic Clouds, M 31 and M 33 galaxies from the 2MASS All-Sky Survey. The 2MASS detection limit allowed us to see only the brightest carbon star candidates in M 31 and M 33. On the other hand, the brightest carbon dust-enshrouded stars are not detected in the 4000-6000 ̊ range in which the published catalogues 
are based for both the SMC and LMC (Kontizas et al. 2001; Rebeirot et al. 1993; Morgan et al. 1995). Our search for the most luminous AGB stars will produce a homogeneous set of data for all four galaxies as confined by the detection limit of 2MASS. Data retrieval and basic analysis were performed through the Virtual Observatory (VO) and relevant tools.

Although the most luminous AGB stars are expected to be carbon stars, a lack of carbon stars is observed for luminosities that are greater than $M_{\text {bol }}=-6.0 \mathrm{mag}$ (van Loon et al. 1991). One explanation is that luminous AGB stars become invisible at wavelengths shorter than $\approx 1 \mu$ m due to obscuration by a circumstellar dust shell as a result of intense mass loss on the TP-AGB (van Loon et al. 1991).

In the LMC cluster HS 327, there is evidence that carbon stars and OH/IR stars may be coeval (van Loon et al. 1991). These stars are most likely $\approx 200$ Myr old and formed at an epoch of intense star formation in the LMC.

Egan et al. (2001), when combining 2MASS and MSX colors, studied the AGB population and other red stars in the LMC, including objects with unusual IR excesses. By using the IR point-source model by Wainscoat et al. (1992) and obtaining source names and spectral types from the SIMBAD database when available, they have identified 11 categories of stellar populations and red nebulae, including main-sequence stars, giant stars, red supergiants (RSGs), C- and O-rich AGB stars, PNs, HII regions, and other dusty objects likely associated with early-type stars. A total of 731 of these sources were previously unidentified. Comparing their results with the bright AGBs of the LMC studied here, we found that the majority of these stars are $\mathrm{OH} / \mathrm{IR}$ and carbon stars.

In this study we provide the catalogues of the brightest AGB stars $\left(-8.4<M_{\text {bol }}<-6.0 \mathrm{mag}\right)$ in four galaxies: the LMC, the SMC, M 31, and M 33. This selection of the magnitude range is due to the limit of detection of 2MASS in M 31 and M 33. The spatial distribution and corresponding luminosity functions are derived and discussed for each galaxy.

\section{Data analysis}

\subsection{VO tools}

The recent developments of the Virtual Observatory creat tools dedicated to some generic operations. Padovani et al. (2004) prove the efficiency of such tools in helping astronomers to produce scientific results using the European Astrophysical Virtual Observatory (AVO). We took advantage of the VO capabilities at various stages of the analysis presented in this paper. For instance, the CDS service Vizier (Ochsenbein et al. 2000) is used as an implicit cross-match tool to identify the LMC carbon stars from Kontizas et al. (2001) in the 2MASS point source catalogue. It is a positional cross-match that looks for sources within a maximum distance chosen by the user ( 1 " for this work). One of the main features of the VO is interoperability. Vizier output for 2MASS queries is directly displayed using VOPlot (Kale et al. 2004), a tool provided by the Indian VO. The interoperability between Vizier and VOPlot is ensured by the exchange of a VOTable ${ }^{1}$ that makes it possible to directly

\footnotetext{
1 http://www.ivoa.net
}

visualize the spatial distribution or color-magnitude diagrams resulting from the catalogue query. Moreover, the filtering capabilities of VOPlot allow one to use criteria to select and display a specific population, such as the AGB stars discussed in this paper. As an example, Fig. 8 shows the superpositon of a color-filtered catalogue on the M 31 GALEX image (Thilker et al. 2005). No WCS (World Coordinate System) information was initially available, but a registration could be obtained using Aladin (Bonnarel et al. 2000), which was the basis of the AVO prototype. There are still limitations in the VO tools. At the moment, for instance, very large catalogues cannot be handled in the same way as small catalogues. While the M 31 catalogue $\left(10^{4}\right.$ sources $)$ is directly output in VOPlot, the LMC catalogue ( 2 million sources) is obtained via vizquery ${ }^{2}$, which allows one to query VizieR remotely for further analysis.

\subsection{AGB stars and bolometric correction}

The data used in this investigation were selected from the 2MASS all-sky survey catalogue restricted to objects for which the error is lower than 0.15 mag in all bands.

There are several criteria for detecting carbon stars in the various wavelengths. For $J H K_{\mathrm{s}}$ filters the most conservative criteria are those by Davidge (2003), who found that stars with $H-K_{\mathrm{s}}>0.4 \mathrm{mag}$ and $J-K_{\mathrm{s}}>1.5 \mathrm{mag}$ in the galaxy NGC 205 are carbon stars. These color limits are known to be metallicitydependent, with $J-K_{\mathrm{s}}$ becoming redder by $0.1 \mathrm{mag}$ when the metallicity changes from $Z=0.004$ to $Z=0.2$ (Davidge 2003). The metallicities of the four galaxies studied here are either similar or lower than the one studied by Davidge, which implies that the color $J-K_{\mathrm{S}}$ can be bluer than the $1.5 \mathrm{mag}$ value adopted here. If taking the metallicity effect into consideration, our sample might not include some of the bluest stars. Considering that the upper part of the red tail contains $\mathrm{C}$-rich, O-rich, OH/IR stars, only spectra would be able to solve the ambiguity about the nature of these bright red stars.

To compare the results found here with previous studies of the carbon star populations in galaxies, the $K_{\mathrm{s}}$ magnitudes of the stars were converted into bolometric magnitudes. The bolometric correction used is an approximation proposed by Bessel \& Wood (1984). Some more recent references are actually based on a similar assumption. Bessel \& Wood (1984) computed the bolometric correction, $B C_{K}$, in the $K$ magnitude for various AGB stars in the Galaxy, LMC, SMC, and 47 Tuc. The available carbon star observations were too few to deduce a relation for them alone, but it was found that the O-rich stars' relation for $M_{\text {bol }}$ describes well the few carbon stars in the $H-K$ vs. $J-K$ diagram. In this same study it was shown that the bolometric corrections vary for stars in galaxies with different metallicities. For stars in the Galaxy and LMC, the correction given by Bessel \& Wood (1984) is $B C_{K}=0.72+2.65 \times$ $(J-K)-0.67 \times(J-K)^{2}$, which was validated for the magnitude range $0.6<J-K<2.0 \mathrm{mag}$. This is the correction we used for the LMC, but also for M 31 and M 33, considering that they have the same metallicity as the Galaxy and LMC respectively. For stars in the SMC, the correction found by

\footnotetext{
${ }^{2}$ http://cdsweb.u-strasbg.fr/doc/vizquery.htx
} 
Bessel \& Wood (1984) was $B C_{K}=0.60+2.65 \times(J-K)-0.67 \times$ $(J-K)^{2}$ for stars with $0.6<J-K<1.5 \mathrm{mag}$. Beyond that color limit no data were available in the SMC, so in this case we used the bolometric corrections for stars in the LMC as an approximation. This might lead to calculated values of $M_{\mathrm{bol}}$ that are higher than the real ones for the SMC stars, but the difference between the two formulae is only $0.1 \mathrm{mag}$. This result is also supported by Montegriffo et al. (1998) who studied the bolometric corrections in several Galactic globular clusters with various metallicities. They found that the bolometric corrections for metal-poor objects are very close to those calculated from the metal-rich relation at redder colors.

\section{Color-magnitude diagrams}

\subsection{The Large Magellanic Cloud}

Modeling the color-magnitude diagram of the red tail of the LMC from the 2MASS data, Marigo et al. (2003) found that field stars are not expected to reach colors that are redder than $J-K_{\mathrm{s}} \approx 1.0 \mathrm{mag}$, thus they hardly contaminate the features produced by the LMC population of AGB stars. We assumed the distance modulus $m-M=18.55 \pm 0.04$ (statistical) \pm 0.08(systematic) mag (Cioni et al. 2004) and the mean reddening $E_{B-V}=0.13$ mag (Massey et al. 1995), which gives an absorption of $A_{K_{\mathrm{s}}}=0.04 \mathrm{mag}$ using $A_{K_{\mathrm{S}}}=0.34 \times E_{B-V}$ (Bessel $\&$ Brett 1988). Carbon star candidates were selected following Davidge criteria (2003) in the 2 MASS $M_{K_{\mathrm{s}}}$ vs. $J-K_{\mathrm{s}}$ diagram presented in Fig. 1. Keeping only objects with 2MASS photometric errors less than $0.15 \mathrm{mag}$, we found 7137 carbon star candidates among the 10055 stars that belong to the red tail, which is defined by $J-K_{\mathrm{s}}>1.3 \mathrm{mag}$ and $M_{K_{\mathrm{s}}}<-6.5 \mathrm{mag}$ (Cioni et al. 2004). The cross-match within a $3^{\prime \prime}$ radius of the 7137 AGB stars with the 7716 spectroscopically confirmed carbon stars from Kontizas et al. (2001) gives 3782 common objects. This confirms that red tail stars contain a large number of carbon stars.

A comparison with Kontizas et al. (2001) data shows that the bright end of the optically detected carbon stars is two magnitudes fainter in the near-infrared than the bright end of the red tail stars detected in 2MASS. The bright AGB stars with $-8.4<M_{\text {bol }}<-6.0 \mathrm{mag}$ are found to be 216 (Table A.1), whereas the total number of the bright red tail stars is 256 . In addition, we searched the DSS catalogue in three colors $B(J)$, $R$, and $I$ to confirm that these stars are not observable in the $B$, $R$ wavelengths. We found that many stars (almost 1 out of 5) are not visible in $B$ and $R$ (traditionally accepted as the representative optical window), but they become detectable or appear very luminous in $I$, as shown in the example of Fig. 2. Deep surveys may reveal these stars, but available observations have not detected them yet.

The spatial distribution of the 7137 red tail AGB stars extracted from 2MASS is presented at the top of Fig. 3, and the bottom figure shows the distribution of the 216 most luminous of them. Figure 3 shows high density in the central regions for the red tail AGB stars, including the most luminous ones, in agreement with previous studies by Hughes \& Wood (1990) and Wood et al. (1985).

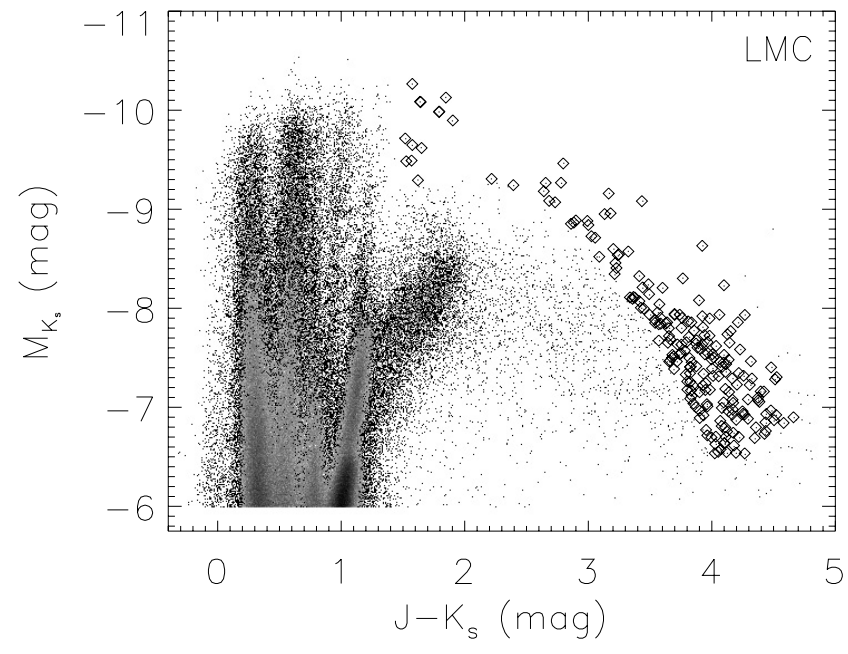

Fig. 1. Color-magnitude diagram for the LMC stars with $M_{K_{\mathrm{s}}}<$ $-6.0 \mathrm{mag}$. The red tail is defined by $J-K_{\mathrm{s}}>1.3 \mathrm{mag}$ and $M_{K_{\mathrm{s}}}<-6.5 \mathrm{mag}$, whereas the luminous AGB stars with $-8.4<$ $M_{\text {bol }}<-6.0$ mag are shown with diamonds.
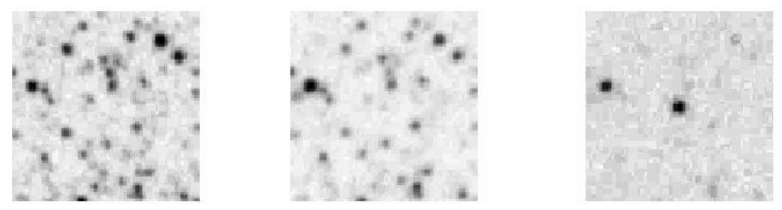

Fig. 2. The $B(J), R, I$ images from the DSS survey of one LMC carbon star detected here (2MASS J04532183-7051449) show that it is only detected in the $I$ band.

Egan et al. (2001) combined LMC data from 2MASS and MSX to detect carbon stars. They defined carbon stars by using the criteria $1.5<K_{\mathrm{s}}-A<3.75 \mathrm{mag}$ and $0.8<H-K_{\mathrm{s}}<$ $1.5 \mathrm{mag}$ and the $\mathrm{OH} / \mathrm{IR}$ with $J-K_{\mathrm{s}}>3 \mathrm{mag}, K_{\mathrm{s}}-A>3.75 \mathrm{mag}$ and $H-K_{\mathrm{s}}>1.5 \mathrm{mag}$. Cross-matching the stars found here with those of Egan et al. (2001) has shown that 20 of the luminous AGB stars are actually carbon stars, 109 of them are $\mathrm{OH} / \mathrm{IR}$ stars, 5 are planetary nebulae, 1 is oxygen-rich AGB and 1 is carbon-rich AGB star, 24 are of unkown nature, and no match was found for the 56 other stars included in our sample.

\subsection{The Small Magellanic Cloud}

Like the LMC, no significant foreground star contamination is expected at $J-K_{\mathrm{s}}>1.0 \mathrm{mag}$ (Marigo et al. 2003) toward the SMC. The distance modulus is $m-M=18.99 \pm$ 0.03 (statistical) \pm 0.08 (systematic) mag (Cioni et al. 2004). The mean reddening adopted here is the one given by Massey et al. (1995) $E_{B-V}=0.09 \mathrm{mag}$, which corresponds to $A_{K_{\mathrm{s}}}=$ 0.03 mag. The red tail known to be located at $J-K_{\mathrm{s}}>1.2 \mathrm{mag}$ and $M_{K_{\mathrm{s}}}<-7.0 \mathrm{mag}$ can be seen in Fig. 4. It contains 1674 stars in the 2MASS catalogue when restricted to sources with photometric errors smaller than $0.15 \mathrm{mag}$. Among them, 911 are carbon star candidates if assuming the Davidge (2003) criteria. There are 34 bright carbon stars with $-8.4<M_{\text {bol }}<$ -6.0 mag (Table A.2) out of a total of 50 bright sources in the red tail. A $3^{\prime \prime}$ radius cross-match of the 911 carbon star 


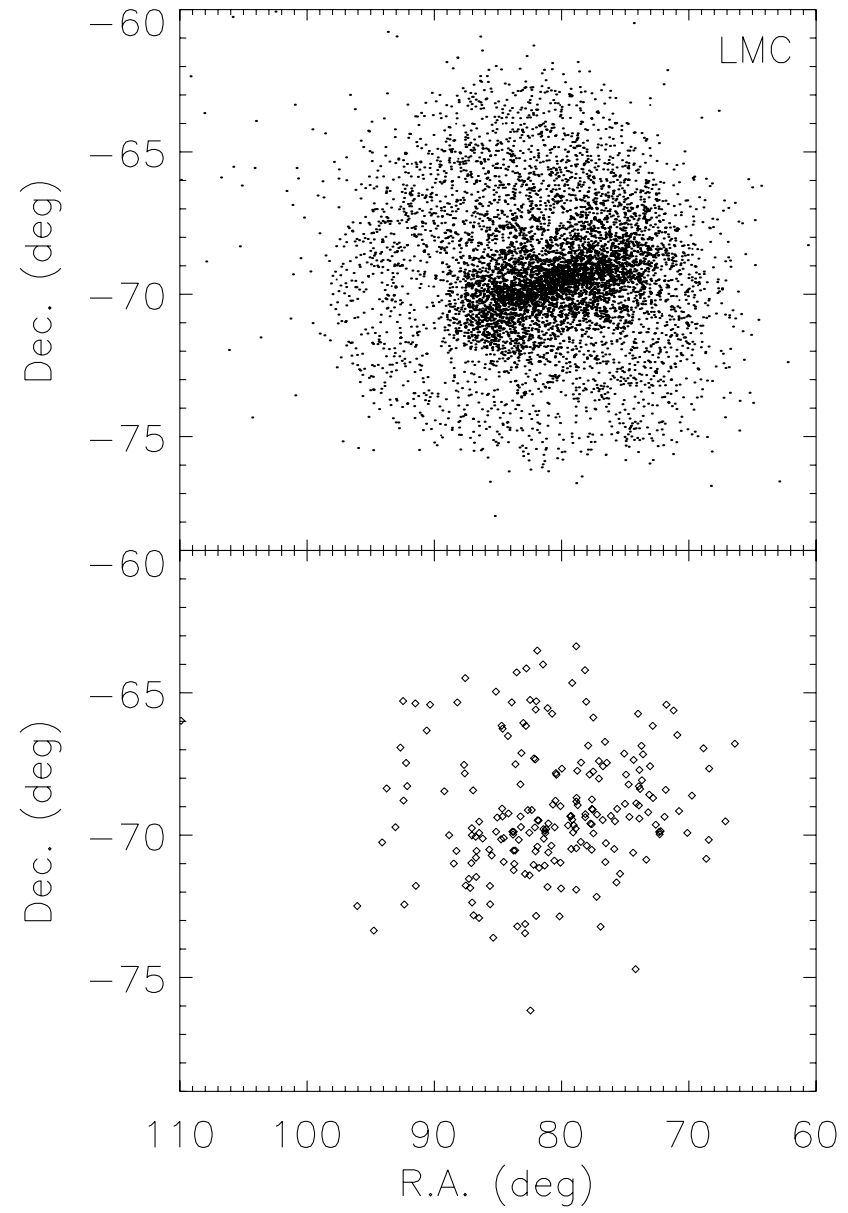

Fig. 3. Spatial distribution of all LMC AGB stars selected in this paper (top), and the very bright ones with $-8.4<M_{\text {bol }}<-6.0 \mathrm{mag}$ (bottom).

candidates with the catalogues of SMC carbon stars confirmed by spectroscopy in the optical wavelengths (Rebeirot et al. 1993; Morgan et al. 1995) gives 676 sources in common. It is worth noting that only one cross-matched star belongs to the 34 very bright $A G B$ stars of our sample.

The carbon star candidates selected here are distributed within an elliptical area, with no obvious central concentration (Fig. 5). The brightest sources with $-8.4<M_{\text {bol }}<-6.0 \mathrm{mag}$ tend to be in the central area, although they are too few in number to draw a definite conclusion. Demers et al. (2003) found that the carbon stars are located almost exclusively in and near the disk for the Magellanic type galaxy NGC 3109. This is consistent with our results in the SMC and the LMC.

\subsection{Messier 31}

M 31 is a more distant galaxy than the LMC and SMC, with a distance modulus of $m-M=24.38 \pm 0.05 \mathrm{mag}$ (Brewer $\&$ Richer 1995). The color-magnitude diagram presented in Fig. 6 shows that only the upper part of the red tail is observable in M 31 because of the 2MASS sensitivity limit. It was assumed that all $J-K_{\mathrm{s}} \geq 1.1 \mathrm{mag}$ stars are red tail stars (see Fig. 6). In that area there are 959 stars with photometric errors less than $0.15 \mathrm{mag}$. Since M 31 is located at an intermediate

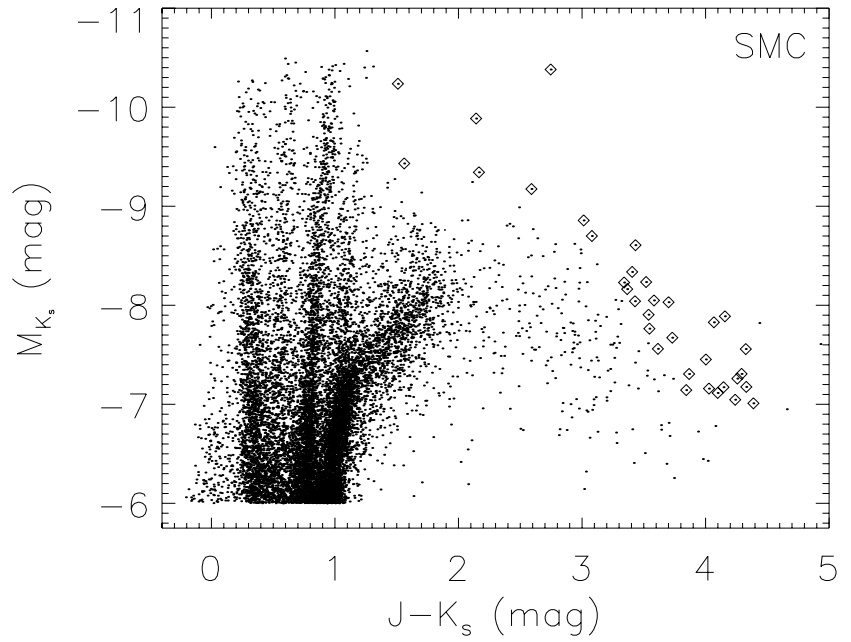

Fig. 4. Color-magnitude diagram for all SMC stars with $M_{K_{\mathrm{S}}}<$ $-6.0 \mathrm{mag}$. The red tail is defined by $J-K_{\mathrm{S}}>1.2 \mathrm{mag}$ and $M_{K_{\mathrm{s}}}<-7.0 \mathrm{mag}$, whereas the luminous ABG stars with $-8.4<$ $M_{\text {bol }}<-6.0$ mag are shown with diamonds.

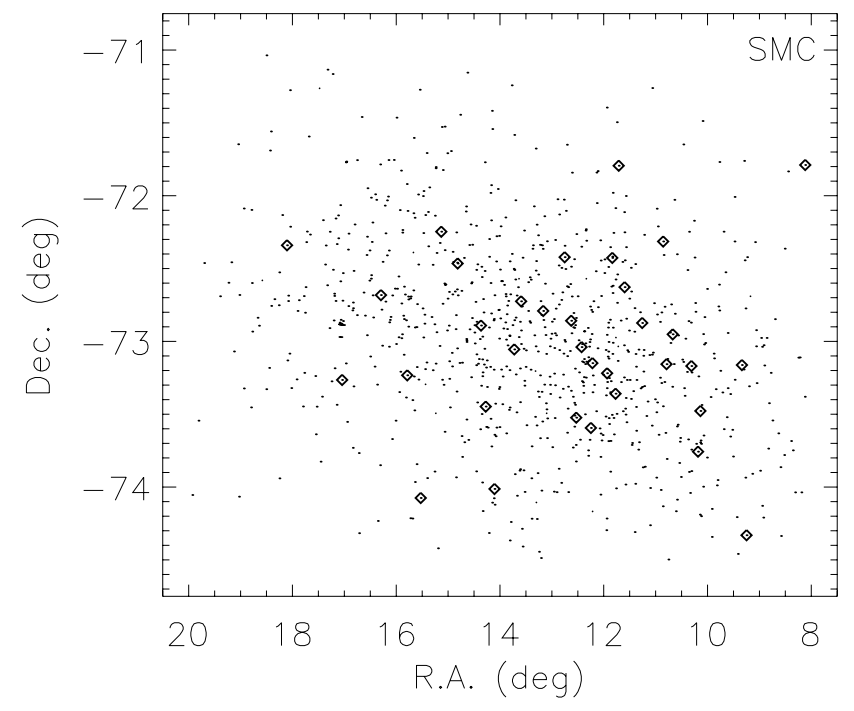

Fig. 5. Spatial distribution of all SMC AGB stars selected in this paper (dots). The most luminous with $-8.4<M_{\text {bol }}<-6.0$ mag are represented with diamonds.

galactic latitude, the question of foreground star contamination needs to be properly addressed. We retrieved data from a region near M 31 (Fig. 7), located at $16.0<$ RA $<17.5$ deg and $40.5<\mathrm{Dec}<42.0 \mathrm{deg}$. The color-magnitude diagram for M 31 is very populated at $J-K_{\mathrm{s}}>1.1 \mathrm{mag}$ (Fig. 6), whereas the neighboring field (Fig. 7) contains very few stars in this part of the diagram, indicating that the contamination from the Milky Way stellar population is very low.

For the total reddening correction near $\mathrm{M} 31, E_{B-V} \approx$ $0.23 \mathrm{mag}$ is adopted (Brewer \& Richer 1995). Consequently the absorption at $K_{\mathrm{s}}$ is $0.08 \mathrm{mag}$, assuming again $A_{K_{\mathrm{s}}}=0.34 \times$ $E_{B-V}$ (Bessel \& Brett 1988). From these relatively low absorption values, we excluded the possibility that the 959 red tail stars are early type stars heavily obscured, although they are located in the spiral structure of M 31. 


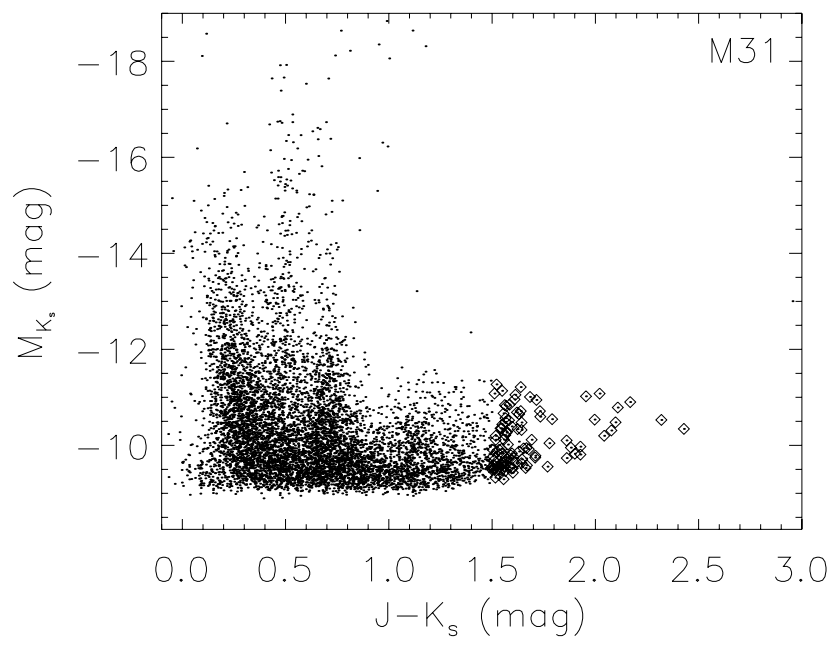

Fig. 6. Color-magnitude diagram for all $\mathrm{M} 31$ stars detected by 2MASS. The upper part of the red tail is located at $J-K_{\mathrm{s}}>1.1 \mathrm{mag}$, whereas the luminous AGB stars with $-8.4<M_{\text {bol }}<-6.0$ mag are shown with diamonds.

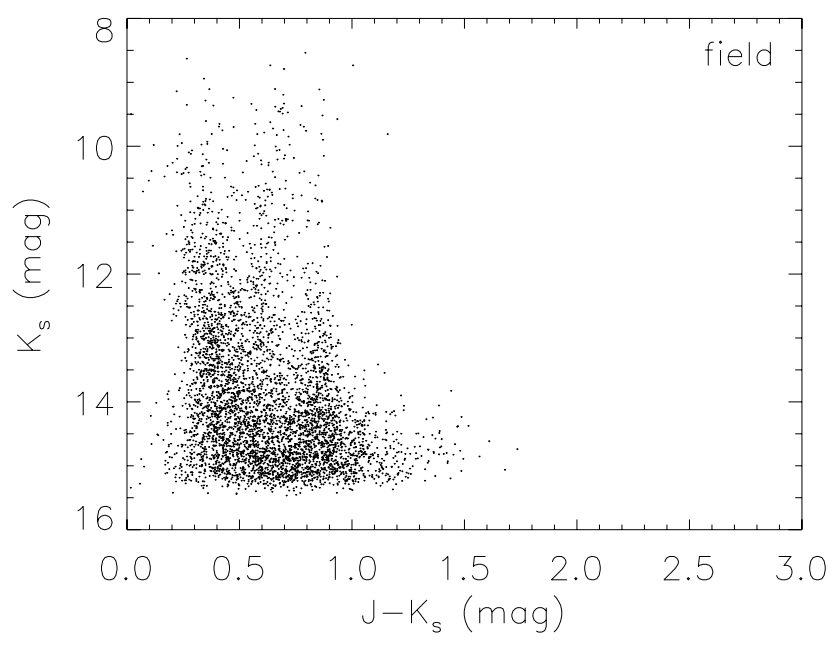

Fig. 7. Color-magnitude diagram for stars detected by 2MASS in a nearby field.

Considering the criteria of Davidge (2003), only 100 stars are carbon star candidates. In Fig. 8 we overplotted the carbon star candidates found in M 31 on the GALEX image. These stars fall on the star forming regions detected at UV wavelengths, following the familiar M 31 ring-structure, $10 \mathrm{kpc}$ away from the galaxy center. This ring has been detected in most of the large scale maps at radio wavelengths (Beck \& Gräve 1982; Brinks \& Shane 1984), and is also traced by starforming regions (Pellet et al. 1978; Devereux et al. 1994). This structure can also be noted in the infrared (Haas et al. 1998), in the optical via masking (Walterbos \& Kennicut 1988), and in the distribution of HII regions (Pellet et al. 1978), OB associations (van der Bergh 1991), HI gas (Sofue \& Kato 1981), and other tracers (see Hodge 1992). The spatial distribution of the carbon star candidates suggests that the velocity dispersion and the differential galactic rotation have not had enough time to spread these bright AGB stars, which are among the youngest intermediate mass stars produced in spiral arms, like the

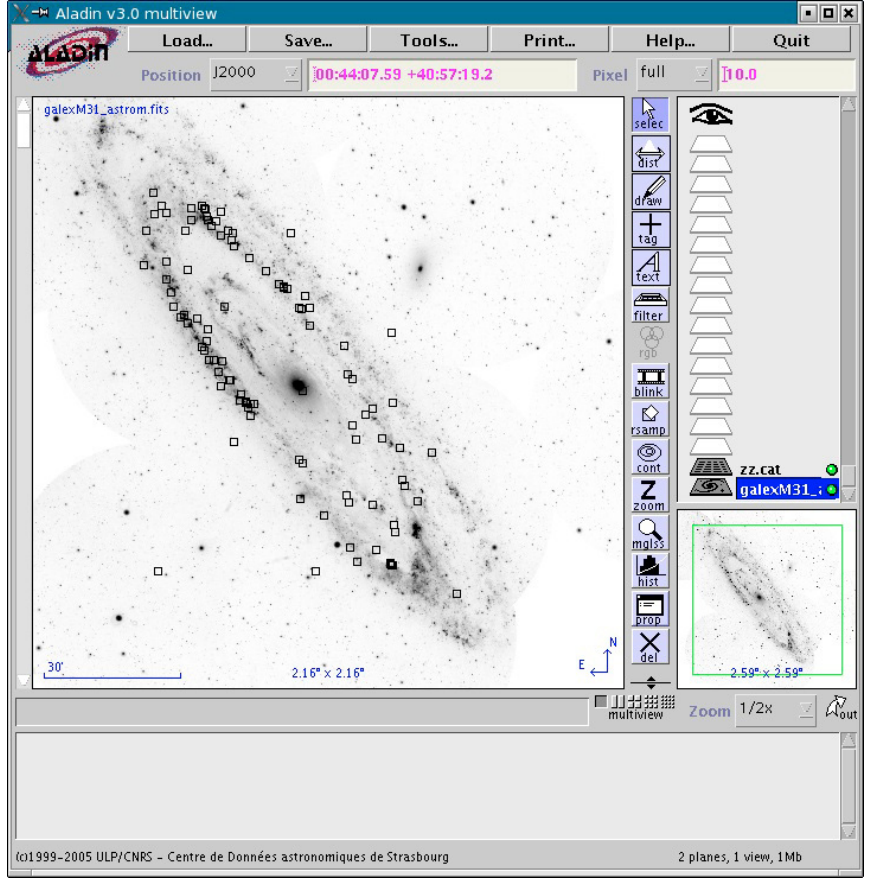

Fig. 8. The brightest carbon star candidates found in M 31 (squares) overplotted on the GALEX UV image, as processed by Aladin v3.0.

current population of young stars. All the 100 bright AGB stars detected here are within the magnitude range of $-8.4<M_{\mathrm{bol}}<$ -6.0 mag. They are listed in Table A.3.

In previous investigations of carbon stars in $M$ 31, either the studied areas are far from the ring of luminous AGB stars presented in this paper or candidate carbon stars are fainter (Brewer et al. 1996; Battinelli et al. 2003, 2005; Davidge et al. 2005).

\subsection{Messier 33}

To complete our survey of the brightest red tail stars in nearby galaxies, we took the 2MASS data for M 33 and followed the same process as for the LMC, SMC, and M 31. Wilson et al. (1991) derive $E_{B-V}=0.3 \pm 0.1$, including both the Milky Way foreground and M 33 internal extinction. This color excess is translated into $A_{K_{\mathrm{s}}} \approx 0.10 \mathrm{mag}$. The distance modulus for M 33 is $m-M=24.52 \pm 0.14$ (statistical) \pm 0.13 (systematic) $\mathrm{mag}$ (Lee et al. 2002), and the red tail is located at $J-K_{\mathrm{s}}>1.0 \mathrm{mag}$ (Fig. 9). Block et al. (2004) consider that stars detected by 2MASS with $J-K_{\mathrm{s}}>1.0$ mag cannot be M-stars in the lowmetallicity regions, indicating the presence of red tail stars. In this area of the color-magnitude diagram, 916 stars have been found; and among them, 31 are supposed to be carbon stars, after we adopt the Davidge (2003) criteria. We stress that all the 31 carbon star candidates are very bright with $-8.4<M_{\text {bol }}<-6.0$ mag (see Table A.4).

The spatial distribution of these stars (Fig. 10) is not correlated with the arcs of red stars found by Block et al. (2004) in the disk of M 33. The cross-identification of these 31 carbon star candidates with those of Rowe et al. (2005) gives no pair within a $1^{\prime \prime}$ search radius. Their study has not revealed the most 


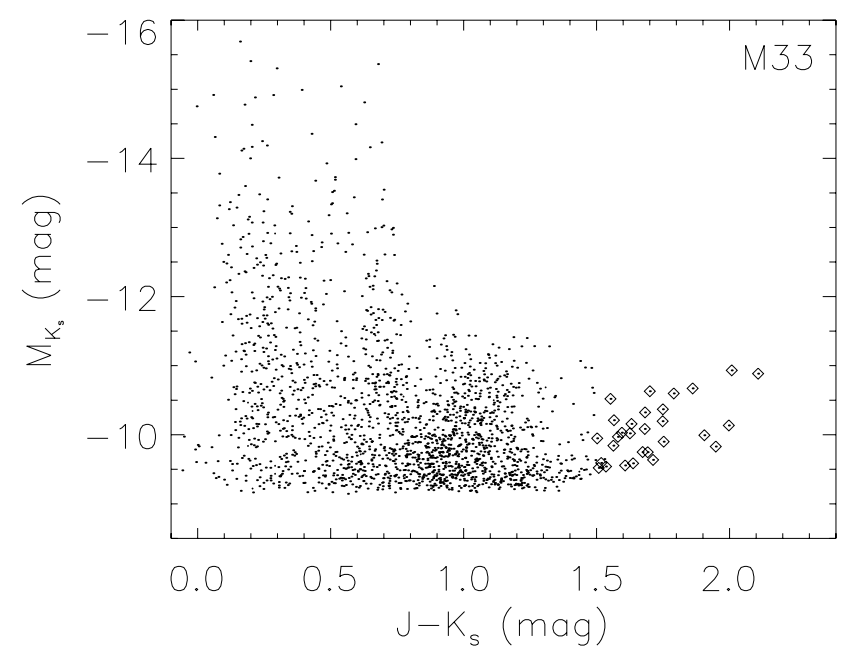

Fig. 9. Color-magnitude diagram for the stars in M 33. The upper part of the red tail is located at $J-K>1.0 \mathrm{mag}$, whereas the luminous AGB stars with $-8.4<M_{\mathrm{bol}}<-6.0 \mathrm{mag}$ are shown with diamonds.

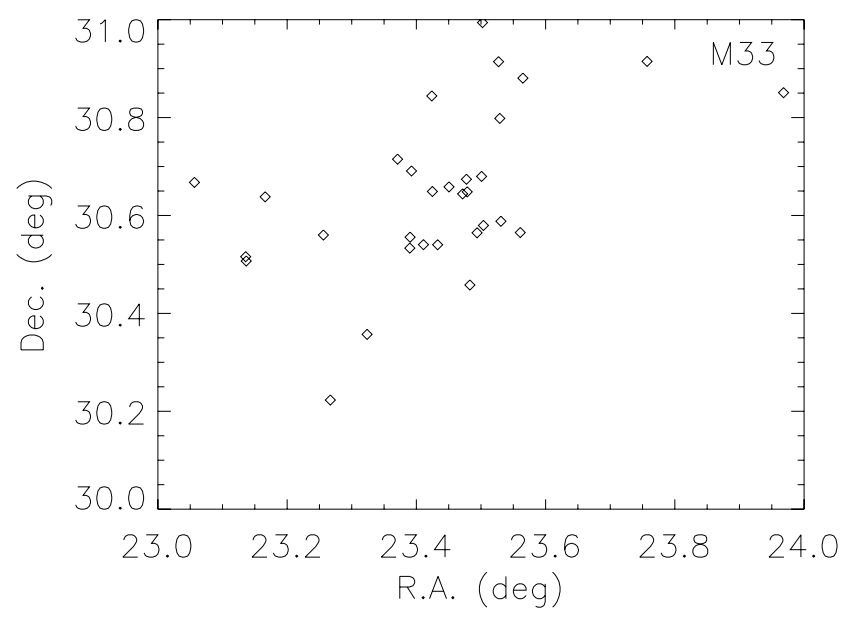

Fig. 10. Spatial distribution of the most luminous AGB stars with $-8.4<M_{\text {bol }}<-6.0$ mag.

luminous AGB star population detected in our paper here, most probably because their observations are in the optical domain where these stars are not detected.

\section{Discussion and conclusion}

\subsection{Luminosity functions}

The luminosity function reflects the distribution of stellar masses formed in a given volume of space for a stellar system or a galaxy. The upper end of the luminosity function displays the most massive stars of any particular population. Carbon stars usually have intermediate mass and provide insight into the star-forming history of these masses.

The luminosity function for the bright AGBs in each galaxy is presented in Fig. 11 (LMC, SMC, M 31, M 33). It illustrates that in all four galaxies, luminous carbon and $\mathrm{OH} / \mathrm{IR}$ candidates (Egan et al. 2001) exhibit a similar luminosity function with slopes varying only slightly and within the errors of the fit. This result suggests that a similar mass distribution is expected

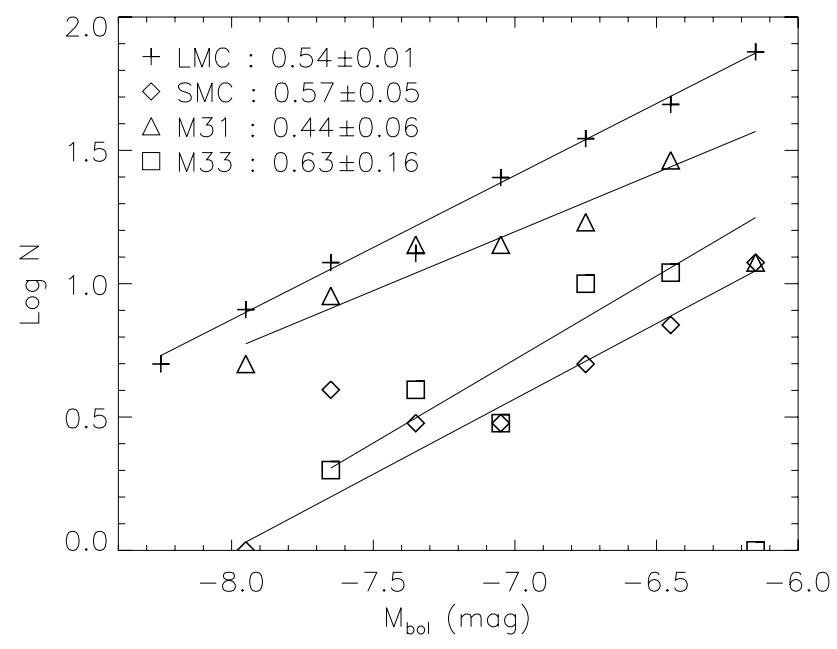

Fig. 11. Luminosity functions for the luminous carbon and $\mathrm{OH} / \mathrm{IR}$ candidates found in the LMC, the SMC, M 31, and M 33. Numbers in the upper-left corner are the slopes of the linear regression fits.

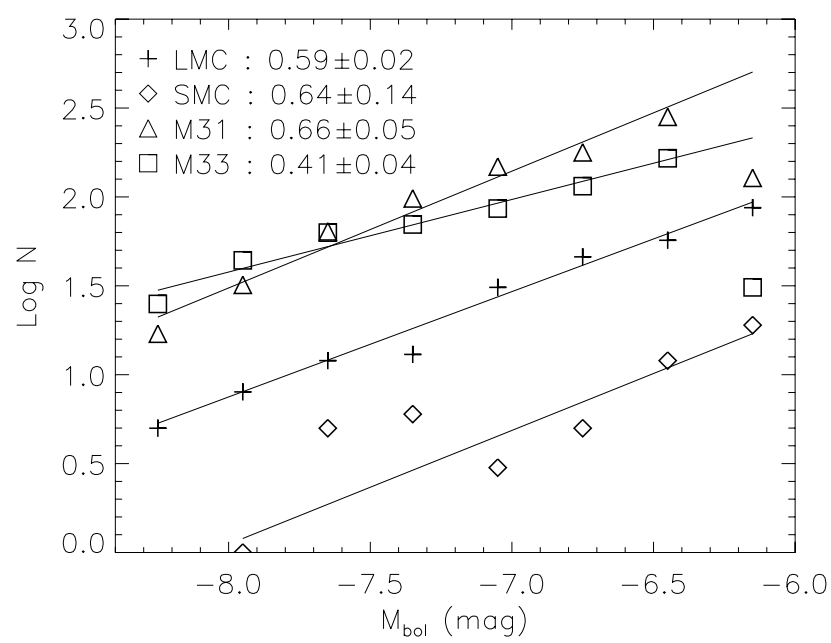

Fig. 12. Luminosity function for the bright red tail stars found in the LMC, the SMC, M 31, and M 33. Numbers in the upper-left corner are the slopes of the linear regression fits.

in all four galaxies for the upper part of the mass function. Groenewegen (2002) found that the faint part of luminosity functions are also similar. Luminosity functions for the bright red tail population are presented in Fig. 12. No significant difference is found between the population of bright $(-8.4<$ $\left.M_{\text {bol }}<-6.0 \mathrm{mag}\right)$ AGB red tail stars $\left(H-K_{\mathrm{s}}>0.4 \mathrm{mag}\right.$ and $J-K_{\mathrm{s}}>1.5 \mathrm{mag}$ ) and the whole population of red tail stars (LMC: $J-K_{\mathrm{s}}>1.3 \mathrm{mag}$ and $M_{K_{\mathrm{s}}}<-6.5 \mathrm{mag}$, SMC: $J-K_{\mathrm{s}}>1.2 \mathrm{mag}$ and $M_{K_{\mathrm{s}}}<-7.0 \mathrm{mag}, \mathrm{M} 31: J-K_{\mathrm{s}}>1.1 \mathrm{mag}$, M 33: $J-K_{\mathrm{s}}>1.0 \mathrm{mag}$ ).

\subsection{The spatial distribution}

In the present work the spatial distribution of the luminous AGB stars appears to depend on the environment. In the LMC they are distributed within an ellipse defined by the extent of the old population of the galaxy. The density is higher along the bar, while the most luminous AGB stars are concentrated 
in the central area where the young cluster systems are located (Kontizas et al. 1993).

In M 31 the AGB stars are distributed on the spiral features of the galaxy. Therefore both the LMC and M 31 show that the luminous and, consequently, the most massive AGB stars trace the location of the star-forming regions of their parent galaxy. On the other hand, the less massive stars that evolve slowly have the time to reach the outer parts of the galaxy, as observed for the LMC. To confirm this behavior in M 31, deeper near-infrared data are required.

In the SMC and M 33, AGBs are located in an elliptical area along the disk, without an obvious higher density at starforming regions, i.e. the center and the spiral structure. It is difficult to draw any conclusion from this diagrams since the amount of luminous AGB stars detected in these galaxies is very small.

\subsection{Conclusion}

We searched for the most luminous AGB stars in four nearby galaxies: the LMC, the SMC, M 31, and M 33. Stars were selected on the red tail of the color-magnitude diagram $\left(M_{K_{\mathrm{s}}}\right.$, $J-K_{\mathrm{s}}$ ) with $J-K_{\mathrm{s}}>1.5 \mathrm{mag}$ and $H-K_{\mathrm{s}}>0.4$ mag. Known optical catalogues of carbon stars in the SMC and LMC do not include these luminous AGB stars, since they were constructed from observations at $B$ and $R$ wavelengths; therefore, the catalogues of luminous AGB stars compiled in this work are a valuable complement to existing data.

Using the same range in $M_{\mathrm{bol}}\left(-8.4<M_{\mathrm{bol}}<-6.0 \mathrm{mag}\right)$ for the four galaxies, we analyzed the spatial distribution of these stars. We found that they follow the spiral ring of M 31 and are mostly located in the central region of the LMC, where all the young population is concentrated. In the SMC and in M 33, their number is too small to draw reliable conclusions about their location. Finally the luminosity functions of the red tail stars and the bright AGB stars, display similar slopes within the same bolometric magnitude range.

Acknowledgements. The authors would like to thank the CDS and the VO for valuable support. P. Tsalmantza and M. Kontizas would like to thank the E.L.K.E. (PYTHAGORAS II) of the University of Athens and the Greek General Secretariat of Research \& Technology. Special thanks go to the University of Strasbourg for financial support and the hospitality of E. \& M. Kontizas. This publication makes use of data products from the Two Micron All Sky Survey, which is a joint project of the University of Massachusetts and the Infrared Processing and Analysis Center/California Institute of Technology, funded by the National Aeronautics and Space Administration and the National Science Foundation. This research made use of the VizieR catalogue access tool and Aladin, CDS, Strasbourg, France. Finally, but not least, we would like to thank the unknown referee for very constructive and stimulating comments.

\section{References}

Battinelli, P., \& Demers, S. 2005, A\&A, 430, 905
Battinelli, P., Demers, S., \& Letarte, B. 2003, AJ, 125, 1298

Beck, R., \& Gräve, R. 1982, A\&A, 105, 192

Bessell, M. S., \& Brett, J. M. 1988, PASP, 100, 1134

Bessell, M. S., \& Wood, P. R. 1984, PASP, 96, 247

Block, D. L., Freeman, K. C., Jarrett, T. H., et al. 2004, A\&A, 425, L37

Bonnarel, F., Fernique, P., Bienaymé, O., et al. 2000, A\&AS, 143, 33

Brewer, J. P., Richer, H. B., \& Crabtree, D. R. 1995, AJ, 109, 2480

Brewer, J. P., Richer, H. B., \& Crabtree, D. R. 1996, AJ, 112, 491

Brinks, E., \& Shane, W. W. 1984, A\&AS, 55, 179

Cioni, M. R. L., Habing, H. J., Loup, C., Epchtein, N., \& Deul, E. 2004, Msngr, 115, 22

Davidge, T. J. 2003, ApJ, 597, 289

Davidge, T. J., Olsen, K. A. G., Blum, R., Stephens, A. W., \& Rigaut, F. 2005, AJ, 129, 201

Demers, S., Battinelli, P., \& Letarte, B. 2003, A\&A, 410, 795

Devereux, N. A., Price, R., Wells, L. A., \& Duric, N. 1994, AJ, 108, 1667

Egan, M. P., Van Dyk, S. D., \& Price, S. D. 2001, AJ, 122, 1844

Frost, C. A., Cannon, R. C., Lattanzio, J. C., Wood, P. R., \& Forestini, M. 1998, A\&A, 332, L17

Groenewegen, M. A. T. 2002 [arXiv: astro-ph/0208449]

Haas, M., Lemke, D., Stickel, M., et al. 1998, A\&A, 338, L33

Hodge, P. 1992, The Andromeda Galaxy (Dordrecht: Kluwer Academic Publishers)

Hughes, S. M. G., \& Wood, P. R. 1990, AJ, 99, 784

Kale, S., Vijayaraman, T. M., Kembhavi, A., et al. 2004, ASP Conf. Ser., 314, 350

Kontizas, E., Dapergolas, A., Morgan, D. H., \& Kontizas, M. 2001, A\&A, 369, 932

Kontizas, M., Kontizas, E., \& Michalitsianos, A. G. 1993, A\&A, 269, 107

Lee, M. G., Kim, M., Sarajedini, A., Geisler, D., \& Gieren, W. 2002, ApJ, 565, 959

Marigo, P., Girardi, L., \& Chiosi, C. 2003, A\&A, 403, 225

Massey, P., Lang, C. C., Degioia-Eastwood, K., \& Garmany, C. D. 1995, ApJ, 438, 188

Montegriffo, P., Ferraro, F. R., Origlia, L., \& Fusi Pecci, F. 1998, MNRAS, 297, 872

Morgan, D. H., \& Hatzidimitriou, D. 1995, A\&AS, 113, 539

Nikolaev, S., \& Weinberg, M. D. 2000, ApJ, 542, 804

Ochsenbein, F., Bauer, P., \& Marcout, J. 2000, A\&AS, 143, 23

Padovani, P., \& AVO 2004, AAS, 20513006

Pellet, A., Astier, N., Viale, A., et al. 1978, A\&AS, 31, 439

Rebeirot, E., Azzopardi, M., \& Westerlund, B. E. 1993, A\&AS, 97, 603

Richer, H. B. 1989, IAU Coll. 106 (Cambridge University Press), 35

Rowe, J. F., Richer, H. B., Brewer, J. P., \& Crabtree, D. R. 2005, AJ, 129, 729

Sofue, Y., \& Kato, T. 1981, Publ. Astron. Soc. Japan, 33, 449

Thilker, D. A., Hoopes, C. G., Bianchi, L., et al. 2005, ApJ, 619, L67

van den Bergh, S. 1991, PASP, 103, 1053

van Loon, J. Th., Zijlstra, A. A., Kaper, L., et al. 2001, A\&A, 368, 239

Wainscoat, R. J., Cohen, M., Volk, K., Walker, H. J., \& Schwartz, D. E. 1992, ApJS, 83, 111

Walterbos, R. A. M., \& Kennicutt, R. C. Jr. 1988, A\&A, 198, 61

Wilson, C. D. 1991, AJ, 101, 1663

Wood, P. R., Bessell, M. S., \& Paltoglou, G. 1985, ApJ, 290, 477 
P. Tsalmantza et al.: Luminous AGB stars in nearby galaxies, Online Material $p 1$

\section{Online Material}


P. Tsalmantza et al.: Luminous AGB stars in nearby galaxies, Online Material p 2

\section{Appendix A: Tables}

Table A.1. Very luminous AGB stars (carbon star candidates) in the LMC.

\begin{tabular}{|c|c|c|c|c|c|}
\hline 2MASS ID & $\begin{array}{r}\text { RA } \\
(\mathrm{deg})\end{array}$ & $\begin{array}{r}\text { Dec } \\
(\mathrm{deg})\end{array}$ & $\begin{array}{r}M_{K} \\
(\mathrm{mag})\end{array}$ & $\begin{array}{r}M_{\mathrm{bol}} \\
(\mathrm{mag})\end{array}$ & $\begin{array}{r}J-K_{\mathrm{s}} \\
(\mathrm{mag})\end{array}$ \\
\hline 2MASS J06103966-6655227 & 92.665289 & -66.922974 & -6.639 & -6.323 & 4.10 \\
\hline 2MASS J06022433-6619263 & 90.601393 & -66.323982 & -7.723 & -7.073 & 3.98 \\
\hline 2MASS J05294752-7609256 & 82.448038 & -76.157127 & -7.677 & -6.752 & 3.88 \\
\hline 2MASS J04564410-7442267 & 74.183760 & -74.707420 & -7.638 & -6.831 & 3.92 \\
\hline 2MASS J06011982-6525021 & 90.332610 & -65.417274 & -7.844 & -6.238 & 3.59 \\
\hline 2MASS J06055819-6522162 & 91.492478 & -65.371185 & -7.392 & -6.798 & 4.00 \\
\hline 2MASS J06094920-6517197 & 92.455038 & -65.288811 & -6.541 & -6.496 & 4.20 \\
\hline 2MASS J05412924-7336038 & 85.371848 & -73.601082 & -7.331 & -6.190 & 3.79 \\
\hline 2MASS J05312948-7326331 & 82.872862 & -73.442551 & -7.010 & -6.314 & 3.96 \\
\hline 2MASS J05074394-7312521 & 76.933107 & -73.214478 & -7.318 & -7.460 & 4.26 \\
\hline 2MASS J05335481-7312143 & 83.478390 & -73.203995 & -6.979 & -6.857 & 4.17 \\
\hline 2MASS J05312710-7307148 & 82.862936 & -73.120804 & -7.485 & -6.878 & 4.00 \\
\hline 2MASS J05455697-7254291 & 86.487392 & -72.908104 & -7.530 & -6.134 & 3.68 \\
\hline 2MASS J05203823-7251062 & 80.159310 & -72.851723 & -7.644 & -6.709 & 3.87 \\
\hline 2MASS J05280053-7250120 & 82.002241 & -72.836685 & -7.038 & -6.050 & 3.85 \\
\hline 2MASS J05474046-7248485 & 86.918596 & -72.813484 & -6.843 & -8.045 & 4.58 \\
\hline 2MASS J05422827-7225333 & 85.617810 & -72.425926 & -7.652 & -6.173 & 3.64 \\
\hline 2MASS J05480721-7221516 & 87.030047 & -72.364342 & -6.636 & -6.315 & 4.10 \\
\hline 2MASS J05085905-7209546 & 77.246083 & -72.165176 & -7.452 & -7.116 & 4.10 \\
\hline 2MASS J05152480-7154493 & 78.853353 & -71.913696 & -6.927 & -7.090 & 4.26 \\
\hline 2MASS J05200955-7152132 & 80.039798 & -71.870346 & -6.927 & -6.793 & 4.17 \\
\hline 2MASS J05484335-7151165 & 87.180628 & -71.854607 & -7.178 & -6.536 & 3.98 \\
\hline 2MASS J05242503-7149020 & 81.104331 & -71.817238 & -7.547 & -6.862 & 3.97 \\
\hline 2MASS J05423112-7146588 & 85.629679 & -71.783005 & -7.226 & -7.522 & 4.31 \\
\hline 2MASS J05500676-7146026 & 87.528169 & -71.767410 & -7.536 & -6.950 & 4.01 \\
\hline 2MASS J06190234-7321026 & 94.759790 & -73.350746 & -6.923 & -7.918 & 4.52 \\
\hline 2MASS J05024447-7139323 & 75.685300 & -71.658974 & -7.097 & -7.602 & 4.37 \\
\hline 2MASS J05490888-7132069 & 87.287000 & -71.535255 & -9.085 & -6.078 & 2.68 \\
\hline 2MASS J05465088-7128033 & 86.712036 & -71.467598 & -6.871 & -7.805 & 4.50 \\
\hline 2MASS J05300380-7124361 & 82.515852 & -71.410049 & -6.690 & -7.112 & 4.35 \\
\hline 2MASS J05313006-7121448 & 82.875254 & -71.362465 & -7.869 & -6.774 & 3.81 \\
\hline 2MASS J05013788-7121123 & 75.407859 & -71.353432 & -7.305 & -8.313 & 4.53 \\
\hline 2MASS J05350379-7113576 & 83.765795 & -71.232681 & -7.885 & -6.174 & 3.54 \\
\hline 2MASS J05270395-7108591 & 81.766488 & -71.149750 & -7.898 & -7.250 & 3.98 \\
\hline 2MASS J06241389-7229196 & 96.057905 & -72.488785 & -9.307 & -6.005 & 2.22 \\
\hline 2MASS J05251951-7104027 & 81.331302 & -71.067429 & -7.859 & -7.861 & 4.21 \\
\hline 2MASS J06092546-7226037 & 92.356121 & -72.434364 & -7.846 & -6.333 & 3.63 \\
\hline 2MASS J05284817-7102289 & 82.200709 & -71.041374 & -8.713 & -6.151 & 3.06 \\
\hline 2MASS J05345289-7100229 & 83.720388 & -71.006363 & -8.853 & -6.035 & 2.86 \\
\hline 2MASS J05535326-7059475 & 88.471917 & -70.996529 & -7.462 & -6.149 & 3.72 \\
\hline 2MASS J05482201-7058476 & 87.091717 & -70.979904 & -7.802 & -6.577 & 3.75 \\
\hline 2MASS J05202105-7057545 & 80.087720 & -70.965149 & -7.620 & -7.114 & 4.03 \\
\hline 2MASS J05061466-7056427 & 76.561102 & -70.945198 & -6.701 & -6.724 & 4.22 \\
\hline 2MASS J05381238-7056174 & 84.551612 & -70.938194 & -6.967 & -6.109 & 3.90 \\
\hline 2MASS J05221654-7053460 & 80.568919 & -70.896126 & -7.635 & -6.332 & 3.72 \\
\hline
\end{tabular}


Table A.1. continued.

\begin{tabular}{|c|c|c|c|c|c|}
\hline 2MASS ID & $\begin{array}{r}\text { RA } \\
(\mathrm{deg}) \\
\end{array}$ & $\begin{array}{r}\text { Dec } \\
(\mathrm{deg}) \\
\end{array}$ & $\begin{array}{r}M_{K} \\
(\mathrm{mag}) \\
\end{array}$ & $\begin{array}{r}M_{\mathrm{bol}} \\
(\mathrm{mag})\end{array}$ & $\begin{array}{r}J-K_{\mathrm{s}} \\
(\mathrm{mag})\end{array}$ \\
\hline 2MASS J04532183-7051449 & 73.340999 & -70.862488 & -7.933 & -8.105 & 4.27 \\
\hline 2MASS J04343118-7049491 & 68.629954 & -70.830322 & -6.548 & -6.278 & 4.12 \\
\hline 2MASS J05465510-7047254 & 86.729615 & -70.790390 & -7.808 & -7.017 & 3.93 \\
\hline 2MASS J06054883-7146462 & 91.453467 & -71.779518 & -7.313 & -6.865 & 4.06 \\
\hline 2MASS J05415782-7042418 & 85.490917 & -70.711617 & -7.710 & -6.627 & 3.81 \\
\hline 2MASS J04573049-7036575 & 74.377079 & -70.615990 & -8.211 & -6.302 & 3.44 \\
\hline 2MASS J05241094-7036210 & 81.045601 & -70.605843 & -8.243 & -6.430 & 3.49 \\
\hline 2MASS J05281148-7033586 & 82.047840 & -70.566299 & -6.704 & -6.192 & 4.03 \\
\hline 2MASS J05530336-7033172 & 88.264027 & -70.554779 & -9.082 & -7.163 & 3.43 \\
\hline 2MASS J05464319-7033154 & 86.679960 & -70.554283 & -7.032 & -6.325 & 3.96 \\
\hline 2MASS J05350751-7032195 & 83.781320 & -70.538757 & -7.798 & -6.337 & 3.65 \\
\hline 2MASS J05343646-7032135 & 83.651938 & -70.537094 & -6.577 & -6.176 & 4.07 \\
\hline 2MASS J05103253-7030497 & 77.635542 & -70.513832 & -8.302 & -7.103 & 3.77 \\
\hline 2MASS J05424425-7030208 & 85.684407 & -70.505783 & -7.175 & -6.434 & 3.95 \\
\hline 2MASS J05032665-7029090 & 75.861058 & -70.485855 & -7.256 & -6.203 & 3.83 \\
\hline 2MASS J05165826-7029085 & 79.242791 & -70.485703 & -8.072 & -6.113 & 3.41 \\
\hline 2MASS J05152170-7027315 & 78.840457 & -70.458771 & -7.904 & -6.295 & 3.59 \\
\hline 2MASS J06162403-7015127 & 94.100149 & -70.253555 & -6.772 & -6.047 & 3.95 \\
\hline 2MASS J05273669-7024036 & 81.902897 & -70.401024 & -7.186 & -6.890 & 4.11 \\
\hline 2MASS J05231239-7022045 & 80.801638 & -70.367935 & -7.657 & -6.528 & 3.79 \\
\hline 2MASS J05120517-7021582 & 78.021575 & -70.366173 & -7.155 & -6.945 & 4.14 \\
\hline 2MASS J06121430-6943119 & 93.059601 & -69.719978 & -6.535 & -6.704 & 4.27 \\
\hline 2MASS J05060423-7016513 & 76.517648 & -70.280930 & -10.087 & -6.823 & 1.64 \\
\hline 2MASS J06094093-6847002 & 92.420551 & -68.783401 & -8.081 & -7.207 & 3.90 \\
\hline 2MASS J05135752-7014104 & 78.489704 & -70.236237 & -7.903 & -6.689 & 3.76 \\
\hline 2MASS J06083184-6816353 & 92.132673 & -68.276482 & -6.743 & -6.633 & 4.17 \\
\hline 2MASS J05332717-7009516 & 83.363228 & -70.164352 & -6.893 & -6.035 & 3.90 \\
\hline 2MASS J04334368-7009504 & 68.432034 & -70.164024 & -6.696 & -6.089 & 4.00 \\
\hline 2MASS J05390173-7008429 & 84.757222 & -70.145256 & -7.737 & -7.490 & 4.13 \\
\hline 2MASS J05253612-7007235 & 81.400528 & -70.123215 & -8.346 & -6.029 & 3.21 \\
\hline 2MASS J05444714-7007047 & 86.196438 & -70.117973 & -7.499 & -6.094 & 3.68 \\
\hline 2MASS J05381127-7006088 & 84.546982 & -70.102470 & -9.649 & -6.417 & 1.58 \\
\hline 2MASS J05470053-7003170 & 86.752248 & -70.054733 & -7.347 & -7.370 & 4.22 \\
\hline 2MASS J05481114-7000167 & 87.046428 & -70.004646 & -6.551 & -6.092 & 4.05 \\
\hline 2MASS J05552103-7000030 & 88.837643 & -70.000847 & -9.463 & -6.573 & 2.80 \\
\hline 2MASS J05351559-6958443 & 83.814974 & -69.978996 & -7.858 & -6.365 & 3.64 \\
\hline 2MASS J04491008-6958048 & 72.292016 & -69.968002 & -7.674 & -6.033 & 3.57 \\
\hline 2MASS J05095999-6956097 & 77.499982 & -69.936028 & -8.574 & -6.449 & 3.32 \\
\hline 2MASS J05250941-6955407 & 81.289231 & -69.927994 & -8.887 & -6.116 & 2.90 \\
\hline 2MASS J05455416-6955172 & 86.475667 & -69.921448 & -7.997 & -6.108 & 3.45 \\
\hline 2MASS J04402848-6955135 & 70.118687 & -69.920441 & -9.897 & -6.560 & 1.90 \\
\hline 2MASS J05300859-6954496 & 82.535810 & -69.913795 & -8.537 & -6.263 & 3.24 \\
\hline 2MASS J05162261-6954181 & 79.094245 & -69.905037 & -7.687 & -6.250 & 3.66 \\
\hline 2MASS J04491848-6953145 & 72.327023 & -69.887367 & -9.159 & -6.764 & 3.17 \\
\hline 2MASS J05403606-6952498 & 85.150270 & -69.880508 & -8.233 & -7.906 & 4.10 \\
\hline 2MASS J05350354-6952454 & 83.764785 & -69.879295 & -7.936 & -7.505 & 4.06 \\
\hline 2MASS J05352686-6952279 & 83.861930 & -69.874420 & -7.536 & -6.787 & 3.94 \\
\hline 2MASS J04485265-6951334 & 72.219410 & -69.859299 & -7.282 & -8.246 & 4.51 \\
\hline
\end{tabular}


Table A.1. continued.

\begin{tabular}{|c|c|c|c|c|c|}
\hline 2MASS ID & $\begin{array}{r}\text { RA } \\
(\operatorname{deg})\end{array}$ & $\begin{array}{r}\text { Dec } \\
(\mathrm{deg})\end{array}$ & $\begin{array}{r}M_{K} \\
(\mathrm{mag})\end{array}$ & $\begin{array}{r}M_{\text {bol }} \\
(\mathrm{mag})\end{array}$ & $\begin{array}{r}J-K_{\mathrm{s}} \\
(\mathrm{mag})\end{array}$ \\
\hline 2MASS J05251232-6950377 & 81.301366 & -69.843819 & -8.325 & -6.364 & 3.41 \\
\hline 2MASS J05254237-6947129 & 81.426567 & -69.786942 & -7.170 & -6.132 & 3.83 \\
\hline 2MASS J05245135-6947042 & 81.213967 & -69.784508 & -8.109 & -6.079 & 3.38 \\
\hline 2MASS J05153398-6945590 & 78.891600 & -69.766396 & -7.161 & -7.737 & 4.40 \\
\hline 2MASS J05481349-6945115 & 87.056240 & -69.753204 & -7.229 & -7.208 & 4.20 \\
\hline 2MASS J05282342-6943387 & 82.097615 & -69.727425 & -6.849 & -7.552 & 4.43 \\
\hline 2MASS J05220985-6943200 & 80.541050 & -69.722244 & -7.934 & -6.580 & 3.70 \\
\hline 2MASS J05324622-6942497 & 83.192614 & -69.713829 & -8.458 & -6.152 & 3.22 \\
\hline 2MASS J05175887-6939231 & 79.495332 & -69.656433 & -9.485 & -6.281 & 1.53 \\
\hline 2MASS J05161189-6938596 & 79.049581 & -69.649895 & -7.222 & -6.224 & 3.85 \\
\hline 2MASS J04502338-6937567 & 72.597436 & -69.632431 & -7.458 & -7.046 & 4.07 \\
\hline 2MASS J05103243-6936542 & 77.635141 & -69.615059 & -7.572 & -6.414 & 3.78 \\
\hline 2MASS J05105680-6935303 & 77.736668 & -69.591759 & -7.584 & -7.638 & 4.23 \\
\hline 2MASS J05240917-6935112 & 81.038249 & -69.586456 & -9.071 & -6.111 & 2.73 \\
\hline 2MASS J05455384-6931233 & 86.474335 & -69.523140 & -9.492 & -6.263 & 1.57 \\
\hline 2MASS J04283018-6930502 & 67.125786 & -69.513962 & -6.732 & -7.409 & 4.43 \\
\hline 2MASS J05031358-6930326 & 75.806610 & -69.509079 & -7.471 & -6.030 & 3.66 \\
\hline 2MASS J05271416-6929112 & 81.809034 & -69.486465 & -6.920 & -6.140 & 3.93 \\
\hline 2MASS J05273189-6928353 & 81.882880 & -69.476494 & -6.614 & -6.521 & 4.18 \\
\hline 2MASS J05070471-6928053 & 76.769657 & -69.468163 & -7.720 & -6.261 & 3.65 \\
\hline 2MASS J05163771-6927142 & 79.157138 & -69.453964 & -7.160 & -7.821 & 4.42 \\
\hline 2MASS J04553354-6924593 & 73.889761 & -69.416473 & -10.266 & -7.034 & 1.58 \\
\hline 2MASS J05401333-6922464 & 85.055542 & -69.379578 & -9.988 & -6.670 & 1.79 \\
\hline 2MASS J05123400-6922086 & 78.141669 & -69.369057 & -7.455 & -6.432 & 3.84 \\
\hline 2MASS J04584149-6921314 & 74.672881 & -69.358727 & -6.960 & -7.074 & 4.25 \\
\hline 2MASS J04473884-6921170 & 71.911845 & -69.354729 & -8.041 & -6.411 & 3.58 \\
\hline 2MASS J06145902-6821262 & 93.745929 & -68.357285 & -7.088 & -6.035 & 3.83 \\
\hline 2MASS J05383397-6920317 & 84.641553 & -69.342140 & -10.082 & -6.817 & 1.64 \\
\hline 2MASS J05171633-6920298 & 79.318059 & -69.341614 & -8.005 & -6.071 & 3.43 \\
\hline 2MASS J05325547-6920266 & 83.231141 & -69.340736 & -6.864 & -6.452 & 4.07 \\
\hline 2MASS J05170066-6919304 & 79.252753 & -69.325127 & -7.770 & -7.598 & 4.15 \\
\hline 2MASS J05042995-6919235 & 76.124802 & -69.323212 & -7.291 & -7.154 & 4.16 \\
\hline 2MASS J05085218-6916337 & 77.217443 & -69.276039 & -7.114 & -6.088 & 3.84 \\
\hline 2MASS J05123206-6915404 & 78.133621 & -69.261230 & -7.602 & -6.697 & 3.88 \\
\hline 2MASS J05364101-6914064 & 84.170893 & -69.235138 & -9.293 & -6.038 & 1.62 \\
\hline 2MASS J04524566-6911494 & 73.190282 & -69.197075 & -8.631 & -7.824 & 3.92 \\
\hline 2MASS J04430510-6909128 & 70.771287 & -69.153557 & -7.530 & -7.206 & 4.10 \\
\hline 2MASS J05303638-6907099 & 82.651612 & -69.119438 & -9.715 & -6.516 & 1.52 \\
\hline 2MASS J05292199-6906584 & 82.341629 & -69.116226 & -7.729 & -6.970 & 3.94 \\
\hline 2MASS J05101432-6906103 & 77.559695 & -69.102882 & -7.222 & -6.426 & 3.93 \\
\hline 2MASS J05102724-6904532 & 77.613541 & -69.081467 & -6.994 & -6.619 & 4.08 \\
\hline 2MASS J05023218-6904377 & 75.634084 & -69.077148 & -6.885 & -7.134 & 4.29 \\
\hline 2MASS J05384137-6903540 & 84.672401 & -69.065002 & -7.076 & -6.948 & 4.17 \\
\hline 2MASS J05202143-6858479 & 80.089325 & -68.979973 & -6.628 & -6.105 & 4.03 \\
\hline 2MASS J04554180-6857227 & 73.924175 & -68.956329 & -7.858 & -6.827 & 3.83 \\
\hline 2MASS J05145597-6856464 & 78.733248 & -68.946243 & -6.756 & -6.500 & 4.12 \\
\hline
\end{tabular}


Table A.1. continued.

\begin{tabular}{|c|c|c|c|c|c|}
\hline 2MASS ID & $\begin{array}{r}\text { RA } \\
(\mathrm{deg}) \\
\end{array}$ & $\begin{array}{r}\text { Dec } \\
(\mathrm{deg})\end{array}$ & $\begin{array}{r}M_{K} \\
(\mathrm{mag}) \\
\end{array}$ & $\begin{array}{r}M_{\mathrm{bol}} \\
(\mathrm{mag})\end{array}$ & $\begin{array}{r}J-K_{\mathrm{s}} \\
(\mathrm{mag})\end{array}$ \\
\hline 2MASS J05224292-6855288 & 80.678873 & -68.924683 & -7.653 & -7.455 & 4.14 \\
\hline 2MASS J05000498-6853553 & 75.020789 & -68.898720 & -9.182 & -6.136 & 2.64 \\
\hline 2MASS J04563215-6852510 & 74.133960 & -68.880844 & -7.466 & -7.790 & 4.32 \\
\hline 2MASS J05152176-6849019 & 78.840680 & -68.817215 & -7.409 & -7.039 & 4.08 \\
\hline 2MASS J05215630-6847225 & 80.484594 & -68.789589 & -6.969 & -7.818 & 4.48 \\
\hline 2MASS J05102834-6844313 & 77.618120 & -68.742050 & -7.244 & -6.511 & 3.95 \\
\hline 2MASS J04511548-6841403 & 72.814508 & -68.694534 & -7.498 & -6.430 & 3.82 \\
\hline 2MASS J05151758-6841198 & 78.823286 & -68.688835 & -6.811 & -6.433 & 4.08 \\
\hline 2MASS J04390199-6836322 & 69.758300 & -68.608963 & -7.050 & -6.714 & 4.10 \\
\hline 2MASS J04522647-6834374 & 73.110316 & -68.577080 & -9.619 & -6.350 & 1.65 \\
\hline 2MASS J05565154-6827267 & 89.214751 & -68.457420 & -9.982 & -6.665 & 1.79 \\
\hline 2MASS J05474884-6825336 & 86.953524 & -68.426003 & -8.143 & -6.340 & 3.49 \\
\hline 2MASS J04471609-6824256 & 71.817052 & -68.407120 & -7.936 & -6.677 & 3.74 \\
\hline 2MASS J04552049-6822391 & 73.835412 & -68.377541 & -8.865 & -6.074 & 2.88 \\
\hline 2MASS J04554249-6816542 & 73.927067 & -68.281723 & -7.408 & -6.360 & 3.83 \\
\hline 2MASS J04585546-6813062 & 74.731110 & -68.218407 & -7.537 & -6.276 & 3.74 \\
\hline 2MASS J05325618-6812487 & 83.234091 & -68.213539 & -9.268 & -6.358 & 2.78 \\
\hline 2MASS J04544534-6804146 & 73.688926 & -68.070747 & -8.110 & -6.008 & 3.34 \\
\hline 2MASS J05081566-6800459 & 77.065279 & -68.012764 & -6.536 & -6.002 & 4.02 \\
\hline 2MASS J05213755-6752393 & 80.406488 & -67.877594 & -7.261 & -6.149 & 3.80 \\
\hline 2MASS J04594477-6752208 & 74.936568 & -67.872452 & -8.599 & -6.264 & 3.20 \\
\hline 2MASS J05111047-6752105 & 77.793643 & -67.869591 & -6.900 & -8.385 & 4.66 \\
\hline 2MASS J05502604-6749462 & 87.608526 & -67.829506 & -7.659 & -6.966 & 3.97 \\
\hline 2MASS J05214756-6749118 & 80.448169 & -67.819962 & -8.728 & -6.123 & 3.03 \\
\hline 2MASS J05100440-6745501 & 77.518363 & -67.763939 & -8.521 & -6.009 & 3.09 \\
\hline 2MASS J05150378-6744142 & 78.765788 & -67.737289 & -7.545 & -6.310 & 3.75 \\
\hline 2MASS J04553202-6742310 & 73.883426 & -67.708626 & -7.358 & -7.068 & 4.11 \\
\hline 2MASS J05195418-6739420 & 79.975765 & -67.661674 & -7.001 & -6.063 & 3.87 \\
\hline 2MASS J04333653-6739329 & 68.402242 & -67.659157 & -6.798 & -7.252 & 4.36 \\
\hline 2MASS J05065934-6734453 & 76.747273 & -67.579254 & -8.096 & -6.033 & 3.36 \\
\hline 2MASS J04521072-6734329 & 73.044681 & -67.575813 & -6.701 & -6.414 & 4.11 \\
\hline 2MASS J05503932-6731504 & 87.663860 & -67.530685 & -7.070 & -7.581 & 4.38 \\
\hline 2MASS J05342976-6730257 & 83.624008 & -67.507164 & -7.839 & -6.198 & 3.57 \\
\hline 2MASS J05054710-6727243 & 76.446275 & -67.456772 & -7.404 & -8.260 & 4.48 \\
\hline 2MASS J05135297-6726548 & 78.470730 & -67.448563 & -10.128 & -6.799 & 1.85 \\
\hline 2MASS J05081617-6723506 & 77.067413 & -67.397408 & -7.179 & -6.069 & 3.80 \\
\hline 2MASS J04572171-6721280 & 74.340464 & -67.357780 & -7.387 & -6.021 & 3.69 \\
\hline 2MASS J05281556-6720188 & 82.064839 & -67.338562 & -7.590 & -6.745 & 3.91 \\
\hline 2MASS J05283913-6718089 & 82.163055 & -67.302483 & -6.798 & -6.499 & 4.11 \\
\hline 2MASS J04542493-6709344 & 73.603899 & -67.159569 & -7.291 & -7.640 & 4.32 \\
\hline 2MASS J05001899-6707580 & 75.079159 & -67.132782 & -9.267 & -6.234 & 2.66 \\
\hline 2MASS J05323716-6706564 & 83.154849 & -67.115669 & -7.943 & -6.586 & 3.70 \\
\hline 2MASS J04352409-6656493 & 68.850409 & -66.947029 & -6.874 & -6.835 & 4.20 \\
\hline 2MASS J04545344-6651375 & 73.722698 & -66.860435 & -8.841 & -6.201 & 3.00 \\
\hline 2MASS J05113864-6651098 & 77.911028 & -66.852730 & -7.853 & -6.151 & 3.54 \\
\hline
\end{tabular}


Table A.1. continued.

\begin{tabular}{|c|c|c|c|c|c|}
\hline 2MASS ID & $\begin{array}{r}\text { RA } \\
(\mathrm{deg})\end{array}$ & $\begin{array}{r}\text { Dec } \\
(\mathrm{deg})\end{array}$ & $\begin{array}{r}M_{K} \\
(\mathrm{mag})\end{array}$ & $\begin{array}{r}M_{\text {bol }} \\
(\mathrm{mag})\end{array}$ & $\begin{array}{r}J-K_{\mathrm{s}} \\
(\mathrm{mag})\end{array}$ \\
\hline 2MASS J04253216-6647163 & 66.384027 & -66.787888 & -7.505 & -6.941 & 4.01 \\
\hline 2MASS J05062118-6643161 & 76.588262 & -66.721153 & -6.932 & -7.655 & 4.44 \\
\hline 2MASS J06085255-6727472 & 92.218968 & -67.463135 & -7.407 & -6.352 & 3.82 \\
\hline 2MASS J05365212-6631004 & 84.217194 & -66.516792 & -7.317 & -6.745 & 4.01 \\
\hline 2MASS J04433797-6628577 & 70.908249 & -66.482704 & -7.934 & -6.141 & 3.50 \\
\hline 2MASS J05383208-6615382 & 84.633676 & -66.260620 & -8.882 & -6.231 & 2.99 \\
\hline 2MASS J05311311-6609411 & 82.804646 & -66.161438 & -7.045 & -6.932 & 4.17 \\
\hline 2MASS J04512141-6609328 & 72.839233 & -66.159134 & -7.513 & -6.138 & 3.69 \\
\hline 2MASS J05385059-6609200 & 84.710828 & -66.155571 & -8.543 & -6.284 & 3.25 \\
\hline 2MASS J05320136-6603279 & 83.005668 & -66.057762 & -7.634 & -6.406 & 3.75 \\
\hline 2MASS J05100176-6551565 & 77.507357 & -65.865715 & -7.153 & -6.289 & 3.90 \\
\hline 2MASS J04555834-6544052 & 73.993113 & -65.734795 & -9.244 & -6.019 & 2.39 \\
\hline 2MASS J05225895-6544029 & 80.745639 & -65.734154 & -7.436 & -7.157 & 4.12 \\
\hline 2MASS J04444769-6537072 & 71.198747 & -65.618690 & -7.080 & -7.329 & 4.29 \\
\hline 2MASS J05280808-6535201 & 82.033687 & -65.588928 & -8.207 & -6.633 & 3.60 \\
\hline 2MASS J05242363-6532107 & 81.098476 & -65.536324 & -7.462 & -6.007 & 3.66 \\
\hline 2MASS J04470329-6524563 & 71.763718 & -65.415649 & -6.761 & -7.474 & 4.44 \\
\hline 2MASS J05524672-6520071 & 88.194668 & -65.335320 & -6.935 & -7.064 & 4.25 \\
\hline 2MASS J05353972-6519564 & 83.915505 & -65.332336 & -6.717 & -6.018 & 3.96 \\
\hline 2MASS J05121594-6518431 & 78.066420 & -65.311981 & -7.344 & -6.286 & 3.82 \\
\hline 2MASS J05275737-6517363 & 81.989046 & -65.293442 & -7.775 & -6.799 & 3.86 \\
\hline 2MASS J05295356-6514567 & 82.473182 & -65.249100 & -7.415 & -6.485 & 3.87 \\
\hline 2MASS J05403844-6457245 & 85.160171 & -64.956818 & -7.480 & -7.262 & 4.14 \\
\hline 2MASS J05164004-6439127 & 79.166850 & -64.653542 & -7.921 & -7.117 & 3.92 \\
\hline 2MASS J05501757-6428545 & 87.573216 & -64.481812 & -8.407 & -6.115 & 3.23 \\
\hline 2MASS J05340710-6416479 & 83.529602 & -64.279976 & -7.959 & -6.297 & 3.56 \\
\hline 2MASS J05123875-6412136 & 78.161467 & -64.203796 & -8.960 & -6.589 & 3.18 \\
\hline 2MASS J05310498-6408306 & 82.770769 & -64.141853 & -8.952 & -6.505 & 3.13 \\
\hline 2MASS J05255025-6400090 & 81.459387 & -64.002502 & -8.114 & -6.064 & 3.37 \\
\hline 2MASS J05273859-6330540 & 81.910813 & -63.515015 & -7.055 & -7.599 & 4.39 \\
\hline 2MASS J05152379-6321528 & 78.849158 & -63.364693 & -6.960 & -6.736 & 4.13 \\
\hline 2MASS J07193710-6559010 & 109.904590 & -65.983627 & -7.339 & -6.559 & 3.93 \\
\hline
\end{tabular}


P. Tsalmantza et al.: Luminous AGB stars in nearby galaxies, Online Material $p 7$

Table A.2. Very luminous AGB stars (carbon star candidates) in the SMC.

\begin{tabular}{|c|c|c|c|c|c|}
\hline 2MASS ID & $\begin{array}{r}\text { RA } \\
(\mathrm{deg})\end{array}$ & $\begin{array}{r}\text { Dec } \\
(\mathrm{deg})\end{array}$ & $\begin{array}{r}M_{K} \\
(\mathrm{mag})\end{array}$ & $\begin{array}{r}M_{\text {bol }} \\
(\mathrm{mag})\end{array}$ & $\begin{array}{r}J-K_{\mathrm{s}} \\
(\mathrm{mag})\end{array}$ \\
\hline 2MASS J00365957-7419503 & 9.248213 & -74.330643 & -9.886 & -6.564 & 2.14 \\
\hline 2MASS J01020652-7404291 & 15.527175 & -74.074753 & -8.164 & -6.114 & 3.37 \\
\hline 2MASS J00562548-7400468 & 14.106208 & -74.013023 & -7.764 & -6.071 & 3.55 \\
\hline 2MASS J00404385-7345244 & 10.182728 & -73.756790 & -7.558 & -7.911 & 4.33 \\
\hline 2MASS J00485947-7335387 & 12.247813 & -73.594093 & -7.889 & -7.729 & 4.16 \\
\hline 2MASS J00500719-7331251 & 12.529961 & -73.523666 & -10.381 & -7.437 & 2.75 \\
\hline 2MASS J00403293-7328399 & 10.137230 & -73.477760 & -7.453 & -6.860 & 4.00 \\
\hline 2MASS J00570590-7326518 & 14.274601 & -73.447746 & -8.337 & -6.363 & 3.41 \\
\hline 2MASS J00470552-7321330 & 11.773011 & -73.359169 & -8.700 & -6.173 & 3.08 \\
\hline 2MASS J01081031-7315524 & 17.042983 & -73.264565 & -9.432 & -6.208 & 1.56 \\
\hline 2MASS J01030900-7313583 & 15.787516 & -73.232887 & -7.260 & -7.402 & 4.26 \\
\hline 2MASS J00474454-7313072 & 11.935618 & -73.218681 & -8.607 & -6.685 & 3.43 \\
\hline 2MASS J00411442-7310091 & 10.310122 & -73.169220 & -7.046 & -7.133 & 4.24 \\
\hline 2MASS J00372080-7309447 & 9.336667 & -73.162430 & -7.173 & -6.981 & 4.15 \\
\hline 2MASS J00430955-7309223 & 10.789804 & -73.156219 & -8.230 & -6.134 & 3.34 \\
\hline 2MASS J00485250-7308568 & 12.218778 & -73.149124 & -7.905 & -6.197 & 3.54 \\
\hline 2MASS J00545410-7303181 & 13.725440 & -73.055054 & -8.235 & -6.485 & 3.52 \\
\hline 2MASS J00494271-7302207 & 12.427986 & -73.039093 & -7.144 & -6.136 & 3.84 \\
\hline 2MASS J00424090-7257057 & 10.670455 & -72.951599 & -8.857 & -6.236 & 3.01 \\
\hline 2MASS J00572770-7253279 & 14.365420 & -72.891106 & -7.159 & -6.634 & 4.03 \\
\hline 2MASS J00450214-7252243 & 11.258941 & -72.873428 & -7.828 & -7.414 & 4.07 \\
\hline 2MASS J00503062-7251298 & 12.627616 & -72.858299 & -10.237 & -7.044 & 1.51 \\
\hline 2MASS J00524017-7247276 & 13.167399 & -72.791023 & -9.174 & -6.085 & 2.59 \\
\hline 2MASS J00542228-7243296 & 13.592872 & -72.724907 & -7.307 & -7.554 & 4.29 \\
\hline 2MASS J01051049-7240563 & 16.293740 & -72.682327 & -7.115 & -6.788 & 4.10 \\
\hline 2MASS J00462352-7237397 & 11.598031 & -72.627708 & -7.306 & -6.356 & 3.87 \\
\hline 2MASS J00591577-7227546 & 14.815710 & -72.465179 & -8.049 & -6.434 & 3.58 \\
\hline 2MASS J00472059-7225360 & 11.835832 & -72.426689 & -7.010 & -7.561 & 4.39 \\
\hline 2MASS J00510074-7225185 & 12.753117 & -72.421829 & -8.031 & -6.679 & 3.70 \\
\hline 2MASS J01122496-7220248 & 18.104013 & -72.340225 & -7.176 & -7.542 & 4.33 \\
\hline 2MASS J00432514-7218511 & 10.854768 & -72.314217 & -9.343 & -6.026 & 2.17 \\
\hline 2MASS J01003166-7214489 & 15.131924 & -72.246941 & -8.041 & -6.111 & 3.43 \\
\hline 2MASS J00465078-7147393 & 11.711623 & -71.794250 & -7.560 & -6.015 & 3.61 \\
\hline 2MASS J00322809-7147207 & 8.117061 & -71.789093 & -7.673 & -6.391 & 3.73 \\
\hline
\end{tabular}


Table A.3. Very luminous AGB stars (carbon star candidates) in M 31.

\begin{tabular}{|c|c|c|c|c|c|}
\hline 2MASS ID & $\begin{array}{r}\text { RA } \\
\text { (deg) }\end{array}$ & $\begin{array}{r}\text { Dec } \\
\text { (deg) }\end{array}$ & $\begin{array}{r}M_{K} \\
(\mathrm{mag})\end{array}$ & $\begin{array}{r}M_{\text {bol }} \\
(\mathrm{mag})\end{array}$ & $\begin{array}{r}J-K_{\mathrm{s}} \\
(\mathrm{mag})\end{array}$ \\
\hline 2MASS J00394632+4030280 & 9.943011 & 40.507805 & -10.339 & -7.083 & 1.62 \\
\hline 2MASS J00422751+4035524 & 10.614644 & 40.597908 & -9.834 & -6.591 & 1.60 \\
\hline 2MASS J00452772+4035589 & 11.365515 & 40.599712 & -9.514 & -6.325 & 1.50 \\
\hline 2MASS J00405951+4036490 & 10.247993 & 40.613636 & -11.009 & -7.727 & 1.68 \\
\hline 2MASS J00405912+4036536 & 10.246338 & 40.614906 & -10.819 & -7.591 & 1.57 \\
\hline 2MASS J00410100+4037072 & 10.254204 & 40.618690 & -9.944 & -6.668 & 1.67 \\
\hline 2MASS J00410122+4037321 & 10.255102 & 40.625584 & -10.320 & -7.055 & 1.64 \\
\hline 2MASS J00413945+4037416 & 10.414384 & 40.628242 & -9.646 & -6.392 & 1.62 \\
\hline 2MASS J00411799+4040170 & 10.324980 & 40.671394 & -10.198 & -6.999 & 1.52 \\
\hline 2MASS J00414753+4040588 & 10.448079 & 40.683018 & -9.645 & -6.429 & 1.55 \\
\hline 2MASS J00405497+4044108 & 10.229067 & 40.736359 & -10.713 & -7.413 & 1.73 \\
\hline 2MASS J00405619+4045461 & 10.234160 & 40.762817 & -11.136 & -7.919 & 1.55 \\
\hline 2MASS J00421697+4047487 & 10.570731 & 40.796864 & -10.310 & -6.976 & 2.08 \\
\hline 2MASS J00412184+4050090 & 10.341023 & 40.835857 & -10.957 & -7.708 & 1.61 \\
\hline 2MASS J00414909+4050485 & 10.454579 & 40.846828 & -9.736 & -6.405 & 1.86 \\
\hline 2MASS J00402901+4050485 & 10.120903 & 40.846828 & -9.907 & -6.717 & 1.50 \\
\hline 2MASS J00424499+4051349 & 10.687467 & 40.859707 & -10.687 & -7.432 & 1.62 \\
\hline 2MASS J00415135+4052139 & 10.463964 & 40.870552 & -9.523 & -6.315 & 1.53 \\
\hline 2MASS J00404426+4054039 & 10.184426 & 40.901096 & -9.651 & -6.452 & 1.52 \\
\hline 2MASS J00404649+4055220 & 10.193716 & 40.922802 & -10.666 & -7.445 & 1.56 \\
\hline 2MASS J00424067+4059225 & 10.669477 & 40.989601 & -9.542 & -6.306 & 1.58 \\
\hline 2MASS J00424586+4059583 & 10.691123 & 40.999554 & -10.352 & -7.136 & 1.55 \\
\hline 2MASS J00401171+4101138 & 10.048817 & 41.020523 & -10.045 & -6.731 & 1.78 \\
\hline 2MASS J00404879+4102304 & 10.203307 & 41.041782 & -9.769 & -6.579 & 1.50 \\
\hline 2MASS J00440117+4104054 & 11.004878 & 41.068172 & -9.585 & -6.369 & 1.55 \\
\hline 2MASS J00413891+4104262 & 10.412128 & 41.073956 & -10.788 & -7.459 & 2.11 \\
\hline 2MASS J00410868+4104383 & 10.286197 & 41.077316 & -10.902 & -7.586 & 2.17 \\
\hline 2MASS J00414286+4107300 & 10.428614 & 41.125015 & -9.872 & -6.671 & 1.52 \\
\hline 2MASS J00434139+4109384 & 10.922468 & 41.160683 & -9.630 & -6.365 & 1.64 \\
\hline 2MASS J00412777+4109388 & 10.365709 & 41.160801 & -9.681 & -6.456 & 1.56 \\
\hline 2MASS J00412030+4111073 & 10.334590 & 41.185371 & -9.927 & -6.712 & 1.55 \\
\hline 2MASS J00434371+4111258 & 10.932143 & 41.190514 & -10.117 & -6.831 & 1.69 \\
\hline 2MASS J00434135+4112137 & 10.922321 & 41.203815 & -10.265 & -7.036 & 1.57 \\
\hline 2MASS J00433662+4112152 & 10.902587 & 41.204224 & -9.793 & -6.553 & 1.59 \\
\hline 2MASS J00405596+4112152 & 10.233187 & 41.204247 & -9.646 & -6.423 & 1.56 \\
\hline 2MASS J00434617+4112331 & 10.942393 & 41.209213 & -9.952 & -6.618 & 1.88 \\
\hline 2MASS J00434686+4112451 & 10.945257 & 41.212532 & -10.839 & -7.615 & 1.56 \\
\hline 2MASS J00435338+4113016 & 10.972420 & 41.217136 & -11.022 & -7.682 & 1.95 \\
\hline 2MASS J00435217+4114228 & 10.967414 & 41.239681 & -9.583 & -6.307 & 1.67 \\
\hline 2MASS J00424061+4115009 & 10.669212 & 41.250271 & -11.215 & -7.952 & 1.64 \\
\hline 2MASS J00441547+4116071 & 11.064496 & 41.268646 & -9.388 & -6.179 & 1.53 \\
\hline 2MASS J00440575+4117199 & 11.023975 & 41.288864 & -10.343 & -7.139 & 2.43 \\
\hline
\end{tabular}


Table A.3. continued.

\begin{tabular}{|c|c|c|c|c|c|}
\hline 2MASS ID & $\begin{array}{r}\text { RA } \\
\text { (deg) }\end{array}$ & $\begin{array}{r}\text { Dec } \\
\text { (deg) }\end{array}$ & $\begin{array}{r}M_{K} \\
(\mathrm{mag})\end{array}$ & $\begin{array}{r}M_{\text {bol }} \\
(\mathrm{mag})\end{array}$ & $\begin{array}{r}J-K_{\mathrm{s}} \\
(\mathrm{mag})\end{array}$ \\
\hline 2MASS J00440440+4117260 & 11.018359 & 41.290573 & -10.537 & -7.197 & 2.00 \\
\hline 2MASS J00414297+4117300 & 10.429050 & 41.291691 & -10.946 & -7.652 & 1.71 \\
\hline 2MASS J00414817+4118332 & 10.450715 & 41.309223 & -9.873 & -6.613 & 1.63 \\
\hline 2MASS J00441789+4119230 & 11.074565 & 41.323059 & -10.849 & -7.612 & 1.58 \\
\hline 2MASS J00441402+4121420 & 11.058431 & 41.361691 & -10.121 & -6.899 & 1.56 \\
\hline 2MASS J00442322+4121496 & 11.096773 & 41.363789 & -9.974 & -6.635 & 1.93 \\
\hline 2MASS J00442890+4121589 & 11.120435 & 41.366371 & -9.809 & -6.470 & 1.93 \\
\hline 2MASS J00443503+4123585 & 11.145990 & 41.399609 & -9.559 & -6.336 & 1.56 \\
\hline 2MASS J00415182+4124420 & 10.465925 & 41.411690 & -10.568 & -7.342 & 1.56 \\
\hline 2MASS J00443680+4124465 & 11.153358 & 41.412933 & -11.078 & -7.739 & 2.02 \\
\hline 2MASS J00443163+4125579 & 11.131823 & 41.432758 & -9.911 & -6.702 & 1.53 \\
\hline 2MASS J00405722+4127239 & 10.238433 & 41.456665 & -9.710 & -6.468 & 1.59 \\
\hline 2MASS J00443076+4128352 & 11.128191 & 41.476463 & -10.435 & -7.217 & 1.55 \\
\hline 2MASS J00423161+4129146 & 10.631723 & 41.487389 & -10.476 & -7.211 & 1.64 \\
\hline 2MASS J00445416+4129530 & 11.225678 & 41.498062 & -10.214 & -6.989 & 1.56 \\
\hline 2MASS J00444232+4130556 & 11.176369 & 41.515465 & -9.295 & -6.074 & 1.56 \\
\hline 2MASS J00450163+4131148 & 11.256814 & 41.520779 & -11.079 & -7.884 & 1.51 \\
\hline 2MASS J00445845+4131352 & 11.243563 & 41.526451 & -10.523 & -7.288 & 1.58 \\
\hline 2MASS J00424172+4132501 & 10.673849 & 41.547260 & -10.530 & -7.268 & 2.32 \\
\hline 2MASS J00424417+4133040 & 10.684061 & 41.551125 & -10.199 & -6.861 & 2.04 \\
\hline 2MASS J00423133+4133103 & 10.630557 & 41.552876 & -10.480 & -7.149 & 2.10 \\
\hline 2MASS J00451065+4133248 & 11.294379 & 41.556915 & -9.826 & -6.490 & 1.90 \\
\hline 2MASS J00441105+4133354 & 11.046080 & 41.559841 & -10.288 & -7.059 & 1.57 \\
\hline 2MASS J00423774+4135395 & 10.657289 & 41.594326 & -9.745 & -6.453 & 1.71 \\
\hline 2MASS J00451348+4136308 & 11.306202 & 41.608582 & -10.546 & -7.229 & 1.79 \\
\hline 2MASS J00425798+4137099 & 10.741592 & 41.619442 & -9.524 & -6.279 & 1.60 \\
\hline 2MASS J00430247+4137386 & 10.760300 & 41.627399 & -10.649 & -7.389 & 1.63 \\
\hline 2MASS J00430142+4137423 & 10.755923 & 41.628422 & -10.393 & -7.150 & 1.60 \\
\hline 2MASS J00430151+4137551 & 10.756292 & 41.631985 & -10.597 & -7.297 & 1.73 \\
\hline 2MASS J00430776+4138124 & 10.782355 & 41.636780 & -10.488 & -7.261 & 1.57 \\
\hline 2MASS J00451834+4139220 & 11.326427 & 41.656124 & -9.708 & -6.442 & 1.64 \\
\hline 2MASS J00432196+4141126 & 10.841507 & 41.686848 & -9.919 & -6.639 & 1.68 \\
\hline 2MASS J00445355+4141357 & 11.223136 & 41.693253 & -9.497 & -6.279 & 1.55 \\
\hline 2MASS J00454539+4142352 & 11.439165 & 41.709801 & -9.465 & -6.240 & 1.56 \\
\hline 2MASS J00451861+4143201 & 11.327579 & 41.722263 & -10.017 & -6.784 & 1.58 \\
\hline 2MASS J00434203+4144032 & 10.925149 & 41.734238 & -9.785 & -6.493 & 1.71 \\
\hline 2MASS J00435943+4146429 & 10.997627 & 41.778587 & -9.328 & -6.131 & 1.52 \\
\hline 2MASS J00440219+4147493 & 11.009132 & 41.797043 & -10.116 & -6.917 & 1.52 \\
\hline 2MASS J00441497+4148529 & 11.062387 & 41.814697 & -9.513 & -6.319 & 1.51 \\
\hline 2MASS J00425297+4149210 & 10.720725 & 41.822521 & -9.685 & -6.493 & 1.51 \\
\hline 2MASS J00440179+4149236 & 11.007496 & 41.823238 & -9.561 & -6.339 & 1.56 \\
\hline 2MASS J00440685+4149571 & 11.028579 & 41.832550 & -9.483 & -6.294 & 1.50 \\
\hline 2MASS J00445634+4149579 & 11.234758 & 41.832775 & -9.519 & -6.277 & 1.59 \\
\hline 2MASS J00454261+4150058 & 11.427546 & 41.834961 & -9.937 & -6.667 & 1.65 \\
\hline 2MASS J00442653+4150557 & 11.110566 & 41.848827 & -10.102 & -6.771 & 1.86 \\
\hline 2MASS J00442081+4151597 & 11.086717 & 41.866611 & -11.269 & -8.068 & 1.52 \\
\hline 2MASS J00443048+4152231 & 11.127036 & 41.873104 & -10.714 & -7.451 & 1.64 \\
\hline
\end{tabular}


Table A.3. continued.

\begin{tabular}{lrrrrr}
\hline \hline 2MASS ID & $\begin{array}{r}\text { RA } \\
(\mathrm{deg})\end{array}$ & $\begin{array}{r}\text { Dec } \\
(\mathrm{deg})\end{array}$ & $\begin{array}{r}M_{K} \\
(\mathrm{mag})\end{array}$ & $\begin{array}{r}M_{\text {bol }} \\
(\mathrm{mag})\end{array}$ & $\begin{array}{r}J-K_{\mathrm{s}} \\
(\mathrm{mag})\end{array}$ \\
\hline 2MASS J00444915+4152273 & 11.204827 & 41.874271 & -11.053 & -7.802 & 1.61 \\
2MASS J00443153+4153031 & 11.131396 & 41.884197 & -10.354 & -7.142 & 1.54 \\
2MASS J00453263+4153356 & 11.385993 & 41.893234 & -9.550 & -6.362 & 1.50 \\
2MASS J00451817+4153503 & 11.325713 & 41.897324 & -9.580 & -6.387 & 1.51 \\
2MASS J00441502+4154102 & 11.062585 & 41.902859 & -9.431 & -6.186 & 1.60 \\
2MASS J00443323+4154382 & 11.138468 & 41.910625 & -9.529 & -6.255 & 1.66 \\
2MASS J00443446+4154487 & 11.143602 & 41.913532 & -9.557 & -6.246 & 1.77 \\
2MASS J00444960+4154503 & 11.206699 & 41.913990 & -9.482 & -6.281 & 1.52 \\
2MASS J00452458+4155249 & 11.352445 & 41.923588 & -9.749 & -6.522 & 1.57 \\
2MASS J00443676+4155300 & 11.153187 & 41.925011 & -9.749 & -6.515 & 1.58 \\
2MASS J00453432+4158096 & 11.393025 & 41.969357 & -9.835 & -6.617 & 1.55 \\
\hline
\end{tabular}

Table A.4. Very luminous AGB stars (carbon star candidates) in M 33.

\begin{tabular}{|c|c|c|c|c|c|}
\hline 2MASS ID & $\begin{array}{r}\mathrm{RA} \\
(\mathrm{deg})\end{array}$ & $\begin{array}{r}\text { Dec } \\
(\mathrm{deg})\end{array}$ & $\begin{array}{r}M_{K} \\
(\mathrm{mag})\end{array}$ & $\begin{array}{r}M_{\mathrm{bol}} \\
(\mathrm{mag})\end{array}$ & $\begin{array}{r}J-K_{\mathrm{s}} \\
(\mathrm{mag})\end{array}$ \\
\hline 2MASS J01331769+3021253 & 23.323723 & 30.357054 & -9.970 & -6.735 & 1.58 \\
\hline 2MASS J01335587+3027285 & 23.482809 & 30.457930 & -10.630 & -7.341 & 1.70 \\
\hline 2MASS J01330404+3013225 & 23.266844 & 30.222937 & -9.714 & -6.399 & 1.78 \\
\hline 2MASS J01323279+3030250 & 23.136661 & 30.506952 & -9.829 & -6.489 & 1.95 \\
\hline 2MASS J01340022+3040475 & 23.500920 & 30.679874 & -10.369 & -7.064 & 1.75 \\
\hline 2MASS J01335459+3040262 & 23.477486 & 30.673952 & -10.671 & -7.340 & 1.86 \\
\hline 2MASS J01340697+3047539 & 23.529077 & 30.798306 & -10.084 & -6.803 & 1.68 \\
\hline 2MASS J01340090+3034465 & 23.503776 & 30.579611 & -9.948 & -6.759 & 1.50 \\
\hline 2MASS J01333366+3033204 & 23.390251 & 30.555672 & -9.750 & -6.472 & 1.67 \\
\hline 2MASS J01341556+3052499 & 23.564865 & 30.880529 & -10.932 & -7.592 & 2.01 \\
\hline 2MASS J01333418+3041271 & 23.392448 & 30.690870 & -10.598 & -7.281 & 1.79 \\
\hline 2MASS J01335855+3033525 & 23.493967 & 30.564590 & -9.540 & -6.331 & 1.54 \\
\hline 2MASS J01335488+3038537 & 23.478687 & 30.648275 & -9.900 & -6.594 & 1.75 \\
\hline 2MASS J01333862+3032254 & 23.410958 & 30.540401 & -10.196 & -6.891 & 1.75 \\
\hline 2MASS J01334194+3038565 & 23.424778 & 30.649031 & -10.520 & -7.301 & 1.55 \\
\hline 2MASS J01334389+3032246 & 23.432910 & 30.540171 & -10.020 & -6.763 & 1.63 \\
\hline 2MASS J01335316+3038389 & 23.471534 & 30.644152 & -10.157 & -6.897 & 1.63 \\
\hline 2MASS J01333357+3031599 & 23.389879 & 30.533327 & -10.884 & -7.555 & 2.11 \\
\hline 2MASS J01334172+3050390 & 23.423867 & 30.844172 & -9.993 & -6.656 & 1.91 \\
\hline 2MASS J01332901+3042541 & 23.370907 & 30.715054 & -9.587 & -6.324 & 1.64 \\
\hline 2MASS J01340652+3054510 & 23.527172 & 30.914177 & -10.135 & -6.795 & 2.00 \\
\hline 2MASS J01334812+3039301 & 23.450541 & 30.658377 & -9.842 & -6.617 & 1.56 \\
\hline 2MASS J01340055+3059387 & 23.502324 & 30.994099 & -9.748 & -6.462 & 1.69 \\
\hline 2MASS J01341459+3033543 & 23.560816 & 30.565096 & -10.211 & -6.985 & 1.57 \\
\hline 2MASS J01355238+3051030 & 23.968257 & 30.850855 & -9.520 & -6.295 & 1.56 \\
\hline 2MASS J01350167+3054534 & 23.756997 & 30.914835 & -10.324 & -7.042 & 1.68 \\
\hline 2MASS J01340739+3035173 & 23.530827 & 30.588150 & -9.636 & -6.343 & 1.71 \\
\hline 2MASS J01323260+3030567 & 23.135842 & 30.515766 & -9.592 & -6.394 & 1.52 \\
\hline 2MASS J01323983+3038170 & 23.165985 & 30.638075 & -9.556 & -6.308 & 1.61 \\
\hline 2MASS J01330147+3033361 & 23.256127 & 30.560051 & -10.030 & -6.788 & 1.60 \\
\hline 2MASS J01321356+3040033 & 23.056537 & 30.667587 & -9.520 & -6.327 & 1.51 \\
\hline
\end{tabular}

